\title{
Bhargava's Cube and Black Hole Charges
}

\author{
Nabamita Banerjee ${ }^{a}$, Ajit Bhand $^{a}$, Suvankar Dutta ${ }^{a}$, Ashoke Sen $^{b}$, Ranveer Kumar Singh ${ }^{a}$ \\ ${ }^{a}$ Indian Institute of Science Education and Research Bhopal \\ Bhopal bypass, Bhopal 462066, India \\ ${ }^{b}$ Harish-Chandra Research Institute, HBNI \\ Chhatnag Road, Jhusi, Allahabad 211019, India
}

E-mail: nabamita,abhand,suvankar,ranveer@iiserb.ac.in, sen@hri.res.in

\begin{abstract}
Black holes in a class of string compactifications, known as STU models, carry four electric and four magnetic charges. Furthermore a duality group, given by the product of three congruence subgroups of $\mathrm{SL}(2, \mathbb{Z})$, acts on these integer valued charges. By placing these eight charges at the eight corners of a Bhargava cube, we provide a classification of the duality orbits in these theories.
\end{abstract}

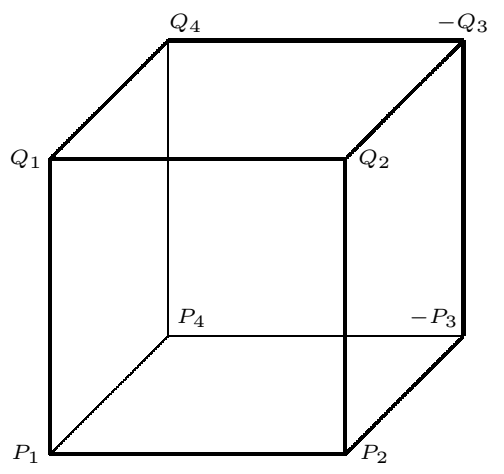




\section{Contents}

1 Introduction $\quad 2$

$2 S L(2, \mathbb{Z})^{3}$ orbits: Primitive case $\quad 7$

\begin{tabular}{ll|l}
3 & $S L(2, \mathbb{Z})^{3}$ orbits: Non-primitive case & 10 \\
\hline
\end{tabular}

4 Orbits of products of congruence subgroups of $S L(2, \mathbb{Z})$

\begin{tabular}{l|l|} 
A Proof of Gauss's Lemma & 25 \\
\hline
\end{tabular}

B Proof of existence of a Bhargava cube for a given pair of quadratic forms 27

\begin{tabular}{ll} 
C Bhargava's results & 30 \\
\hline
\end{tabular}

D Automorphisms of binary quadratic forms

E Possible forms of $\widetilde{S}\left(M_{0}, V\right)$

F Counting duality orbits in different ways

F.1 $D=-16$ and duality group $S L(2, \mathbb{Z})_{S} \times \Gamma_{0}(2)_{T} \times S L(2, \mathbb{Z})_{U} \ldots \ldots . . . . \quad 36$

F.2 $D=-16$ and duality group $\Gamma_{0}(2)_{S} \times S L(2, \mathbb{Z})_{T} \times \Gamma_{0}(2)_{U} \ldots \ldots . . . . .38$

F.3 $D=-36$ and duality group $S L(2, \mathbb{Z})_{S} \times S L(2, \mathbb{Z})_{T} \times \Gamma_{0}(2)_{U} \ldots \ldots . . . .40$

F.4 $D=-12$ and duality group $S L(2, \mathbb{Z})_{S} \times \Gamma_{0}(2)_{T} \times S L(2, \mathbb{Z})_{U} \ldots \ldots . . . .41$

F.5 $D=-12$ and duality group $S L(2, \mathbb{Z})_{S} \times \Gamma_{0}(2)_{T} \times \Gamma_{0}(2)_{U} \ldots \ldots . . . . .42$

\section{Introduction}

The relation between black holes in string theory and class groups was first explored in [13. According to this, there is a one to one correspondence between the duality orbits of supersymmetric black holes in heterotic string theory compactified on a six dimensional torus $T^{6}$ and the equivalence classes of positive definite binary quadratic forms - quadratic forms of the form $A x^{2}+B x y+C y^{2}$, up to equivalence under $S L(2, \mathbb{Z})$ transformations on $(x, y)$. For a given black hole, the constants $A, B$ and $C$ themselves are constructed from T-duality 
invariant quadratic combinations of electric and magnetic charges 1 This relation has been explored in more detail in recent papers [8,9]. In particular, [8] listed several open questions regarding this relation. One of the most interesting of these questions is as follows. We call a binary quadratic form $A x^{2}+B x y+C y^{2}$ primitive if $\operatorname{gcd}(A, B, C)=1$. It is well known that the set of equivalence classes of positive definite primitive binary quadratic forms with a fixed discriminant $D=B^{2}-4 A C$ has the structure of an abelian group known as the class group - given two such equivalence classes, we have a composition rule that produces a third equivalence class of binary quadratic forms with the same discriminant (see e.g. [10,11]). This would suggest that a pair of supersymmetric black holes, with the same discriminant, can be composed to produce a third supersymmetric black hole. The question is: what is the physical interpretation of such compositions?

We shall not try to answer this question. Instead we shall describe a different application of the composition of binary quadratic forms using the description of Bhargava [12]. In this description, which will be described in detail in \$2, one describes the composition with the help of a cube, with integers placed at different corners of the cube. Bhargava's prescription associates a binary quadratic form with every pair of opposite faces of the cube. Since there are three such pairs, we have three such quadratic forms. It can be shown that they all have the same discriminant, and furthermore, that if we apply class group composition on the equivalence classes of these three quadratic forms, the result is the identity element of the group. Therefore Bhargava's prescription gives a new way of describing group composition, by identifying the quadratic form associated with one pair of faces as the inverse of the composition of the quadratic forms associated with the other two pairs of faces.

We shall use Bhargava's construction to address a different problem involving black hole charges in string theory. 2 In a class of string compactifications known as the STU models [13] 18], any state is characterized by a 4 dimensional electric charge vector $Q$ and a 4 dimensional magnetic charge vector $P$, normalized to have integer entries. They are acted upon by the T-duality group $\Gamma_{U} \times \Gamma_{T}$ and the S-duality group $\Gamma_{S}$, where $\Gamma_{U}, \Gamma_{T}$ and $\Gamma_{S}$ are isomorphic to some congruence subgroups of $S L(2, \mathbb{Z})$. The problem we want to address is: given two charge vectors $(Q, P)$ and $\left(Q^{\prime}, P^{\prime}\right)$, how do we know if they are in the same duality orbit, i.e. that they can be related by a $\Gamma_{S} \times \Gamma_{T} \times \Gamma_{U}$ transformation? Unlike in the case of heterotic string

\footnotetext{
${ }^{1}$ This relation holds only for a special class of charges for which the discriminant $D=B^{2}-4 A C$ is either odd and square free, or even and $D / 4$ is square free and is congruent to 2 or 3 modulo 4 . For more general charges the duality orbits require extra data [4-7.

${ }^{2}$ In string theory one does not always distinguish between black holes and other single particle states; our use of the word black hole should be taken in this spirit.
} 
theory on $T^{6}$, where for special class of charges discussed in footnote1, the orbit is classified by the equivalence class of a single quadratic form composed from T-duality invariant quadratic combination of the charges, the answer here is more complicated. We shall show that a compact answer to this question is provided by the Bhargava cube [12], with the eight components of the electric and magnetic charges sitting at the eight corners of the cube. This also provides physical interpretation of the integers sitting at the corners of the Bhargava cube - they are the charges carried by the black holes of the STU model. In other words, each Bhargava cube describes a single black hole in the STU model, unlike in [8,9], where it would correspond to a map from a pair of black holes to a third black hole.

We shall restrict our analysis to charges for which the discriminant $B^{2}-4 A C$ of the associated quadratic forms is negative, since only in this case a single black hole carrying these charges has regular event horizon. This requires $A C>0$. However, we shall not assume that $A$ and $C$ themselves are positive. Therefore the quadratic forms can be either positive definite or negative definite. We shall also not assume that the quadratic forms are primitive. Furthermore, we shall assume that the charges can take arbitrary integer values. In specific models, there may be further duality invariant constraints on the charges that depend on the details of the model. These may involve imposing positive definiteness on some of the quadratic forms, and / or restricting the charges to belong to a sublattice of the integer lattice. This will eliminate some of the duality orbits that we find in our analysis.

The problem of classification of the duality orbits under $\Gamma_{S} \times \Gamma_{T} \times \Gamma_{U}$ could also arise in more general situations, e.g. in the case of heterotic or type II string theories compactified on six dimensional torus $T^{6}$. In these cases the duality groups are much larger, e.g. $O(6,22 ; \mathbb{Z}) \times$ $S L(2, \mathbb{Z})$ for heterotic on $T^{6}$ and $E_{7,7}(\mathbb{Z})$ for type II on $T^{6}$. The duality transformations act simultaneously on the charges and the moduli space of the theory. However we may choose to work in a subspace of the full moduli space of the theory which is invariant under some discrete symmetry group $G$, and examine the spectrum of states carrying definite representation of $G$, or consider twisted index where we count states weighted by the elements of $G$ [19]. In this case only the part of the duality group that commutes with $G$ will be a symmetry of the $G$-twisted index. This part of the duality group can be much smaller and in special cases may have the structure of a product of congruence subgroups of $S L(2, \mathbb{Z})$. Furthermore, questions of this type are well defined only for states carrying charge vectors that are invariant under $G$, and in special cases these could be four dimensional electric and magnetic charges on which the residual duality group acts. A concrete example of this may be provided as follows. One 
possible stringy embedding of the STU model (given in example D in [13]) is to take type II string theory on $T^{4} \times T^{2}$, and take the quotient of the theory by a $\mathbb{Z}_{2} \times \mathbb{Z}_{2}$ discrete symmetry $G$ associated with $T^{4}$ compactification, accompanied by a translation along $T^{2}$. $G$ commutes with only an $S L(2, \mathbb{Z})^{3}$ subgroup of the duality group, and the translation along $T^{2}$ commutes with only a $\Gamma_{0}(2)^{3}$ subgroup of the $S L(2, \mathbb{Z})^{3}$, breaking the duality symmetry to $\Gamma_{0}(2)^{3}$. However, instead of taking the quotient by the discrete symmetry, if we consider the $G$ twisted index in type II on $T^{4} \times T^{2}$ [19], then the relevant symmetry of the twisted index is $S L(2, \mathbb{Z})^{3}$, since in the absence of translation along $T^{2}$ there is no breaking of $S L(2, \mathbb{Z})^{3}$ to $\Gamma_{0}(2)^{3}$. The charge vectors invariant under $G$ are four component electric and four component magnetic charges, on which the residual $S L(2, \mathbb{Z})^{3}$ duality group acts. Therefore in this situation we would require classification of the duality orbits under the $S L(2, \mathbb{Z})_{S} \times S L(2, \mathbb{Z})_{T} \times S L(2, \mathbb{Z})_{U}$ symmetry. Similar analysis can be carried out for other string compactifications whose exact duality group contains as a subgroup product of three congruence subgroups of $S L(2, \mathbb{Z})$, e.g. for the FHSV model [20].

We now give a short summary of our results. In $₫ 2$ we study the duality orbits of $S L(2, \mathbb{Z})_{S} \times$ $S L(2, \mathbb{Z})_{T} \times S L(2, \mathbb{Z})_{U}$. With the eight component charge vector $\left(Q_{1}, \ldots, Q_{4}, P_{1}, \ldots P_{4}\right)$ we can construct three quadratic forms, each being invariant under two of the $S L(2, \mathbb{Z})$ groups and transforming under the third one. Therefore we have three equivalence classes. However by placing the eight components of charges at the eight corners of a Bhargava cube, we show that when the quadratic forms are primitive, i.e. the coefficients $A, B$ and $C$ appearing in each of the quadratic forms are coprime 3 then the equivalence class of the third quadratic form is uniquely determined in terms of the equivalence classes of the other two. In fact, we shall show that the full charge vector $\left(Q_{1}, \ldots, Q_{4}, P_{1}, \ldots P_{4}\right)$ is determined, up to duality transformation, by the equivalence classes of two of the quadratic forms. Therefore the duality orbits are completely classified by equivalence classes of a pair of quadratic forms. In $\$ 3$ we generalize the analysis to the case where the quadratic forms are not primitive. We call a pair of quadratic forms coprime if they do not have a common integer factor. We show that if two of the quadratic forms are coprime, even though they may not be individually primitive, the charge vector is still uniquely determined, up to duality transformation, by the equivalence classes of the first two quadratic forms. Therefore the duality orbits continue to be classified by the equivalence classes of a pair of quadratic forms. When the two quadratic forms are not coprime, but have a common integer factor $m>1$, then the duality orbit is classified by the equivalence classes of the two quadratic forms, the integer $m$, and a pair of integers $(d, b)$, where $d$ is a factor of $m$ and $0 \leq b \leq(d-1)$.

\footnotetext{
${ }^{3}$ If two of the quadratic forms are primitive, then one can show that the third one is automatically primitive.
} 
However, special care is needed if any of the quadratic forms is proportional to either $x^{2}+y^{2}$ or $x^{2}+x y+y^{2}$ up to $S L(2, \mathbb{Z})$ transformation, since these have non-trivial automorphisms $S L(2, \mathbb{Z})$ transformations under which these quadratic forms remain invariant [21,22]. In this case there are additional identifications between the duality orbits labelled by $(a \equiv m / d, b, d)$. In particular if any of the quadratic forms is proportional to $x^{2}+y^{2}$, then $(a, b, d)$ and $\left(a^{\prime}, b^{\prime}, d^{\prime}\right)$ describe the same duality orbit if $a^{\prime}=\operatorname{gcd}(b, d), d^{\prime}=a d / \operatorname{gcd}(b, d)$ and $b^{\prime}$ is related to $b$ in a more complicated fashion explained in (3.25), (3.26). On the other hand if any of the quadratic forms is proportional to $x^{2}+x y+y^{2}$, then $(a, b, d)$ and $\left(a^{\prime}, b^{\prime}, d^{\prime}\right)$ describe the same duality orbit provided $a^{\prime}=\operatorname{gcd}(b, d), d^{\prime}=a d / \operatorname{gcd}(b, d)$, and $b^{\prime}$ is determined by solving (3.29). In this case there is a further identification - the orbits labelled by $(a, b, d)$ and $\left(a^{\prime}, b^{\prime}, d^{\prime}\right)$ are also the same if $a^{\prime}=\operatorname{gcd}(d, a+b), d^{\prime}=a d / \operatorname{gcd}(d, a+b)$, and $b^{\prime}$ is obtained by solving (3.32).

In $\$ 4$ we turn to the problem of classification of the duality orbits of $\Gamma_{S} \times \Gamma_{T} \times \Gamma_{U}$, where $\Gamma_{S}$, $\Gamma_{T}$ and $\Gamma_{U}$ are congruence subgroups of $S L(2, \mathbb{Z})$, but are not necessarily the same congruence subgroups. In this case we need the notion of $\Gamma$ equivalence classes [23] - two quadratic forms are considered equivalent if they are related by a $\Gamma$-transformation, $\Gamma$ being a congruence subgroup of $S L(2, \mathbb{Z})$. As before, we need to pick two of the three quadratic forms associated with the charge vector - for definiteness we take them to be those that transform under $\Gamma_{S}$ and $\Gamma_{U}$. Therefore they are classified by $\Gamma_{S}$ and $\Gamma_{U}$ equivalence classes respectively. We show that if these quadratic forms are coprime, then the duality orbit is classified by their $\Gamma_{S}$ and $\Gamma_{U}$ equivalence classes, and the choice of an element of the coset $\Gamma_{T} \backslash S L(2, \mathbb{Z})$. On the other hand if the two quadratic forms have a common factor $m$, then besides the data described above, and $m$, we also need to choose a pair of integers $(d, b)$, where $d$ is a factor of $m$ and $0 \leq b \leq(d-1)$. Finally for special quadratic forms, related to $\left(x^{2}+y^{2}\right)$ or $\left(x^{2}+x y+y^{2}\right)$ by $S L(2, \mathbb{Z})$ transformation up to a multiplicative constant, some of the duality orbits labelled by $m, b, d$ and the elements of $\Gamma_{T} \backslash S L(2, Z)$, get identified. The procedure for determining when that happens has been explained in $\$ 4$.

In Appendix $\mathrm{A}$ we review the proof of Gauss's Lemma dealing with properties of $2 \times n$ matrices with integer entries. This is well known to mathematicians but may not be so wellknown to physicists. In Appendix $\mathrm{B}$ we describe a proof of the existence of Bhargava's cube for a given pair of binary quadratic forms even when they are not primitive or coprime, generalizing the corresponding result for primitive quadratic forms. For a pair of primitive quadratic forms, the Bhargava cube is known to be unique up to $S L(2, \mathbb{Z})$ transformations, but this is not so when the two quadratic forms are not primitive and not coprime. Appendix C contains a brief 
review of the relevant parts of Bhargava's results that we use in our analysis. Appendix D reviews properties of some special quadratic forms that remain invariant under some $S L(2, \mathbb{Z})$ transformations, since our analysis runs into additional subtleties for such special quadratic forms. Appendix E contains a technical result that is necessary to classify the duality orbits when we have such special quadratic forms. In Appendix $\mathrm{E}$ we count the number of duality orbits in several examples, verifying that the number is invariant under permutations of $\Gamma_{S}$, $\Gamma_{T}$ and $\Gamma_{U}$, even though the algorithm we give treats $\Gamma_{T}$ differently from $\Gamma_{S}$ and $\Gamma_{U}$.

Note added: After submitting the paper to the arXiv, we became aware of Refs. [24, 25] where possible connection between Bhargava's cube and the black hole charges in the STU model was discussed. Although the original references are not available in the arXiv, a review of these results can be found in the recent arXiv paper [26].

\section{$2 S L(2, \mathbb{Z})^{3}$ orbits: Primitive case}

In this section we shall consider the case where the duality group is $S L(2, \mathbb{Z})_{S} \times S L(2, \mathbb{Z})_{T} \times$ $S L(2, \mathbb{Z})_{U}$. We denote the four components of the electric charge vector $Q$ by $Q_{1}, \ldots, Q_{4}$ and the four components of the magnetic charge vector $P$ by $P_{1}, \ldots, P_{4}$. The charges are normalized so that $Q_{i}, P_{i} \in \mathbb{Z}$ for $1 \leq i \leq 4$. Duality transformation by an element of $S L(2, \mathbb{Z})_{S} \times$ $S L(2, \mathbb{Z})_{T} \times S L(2, \mathbb{Z})_{U}$ gives a map $\left(Q_{1}, \ldots, Q_{4}, P_{1}, \ldots, P_{4}\right) \in \mathbb{Z}^{8} \mapsto\left(Q_{1}^{\prime}, \ldots, Q_{4}^{\prime}, P_{1}^{\prime}, \ldots, P_{4}^{\prime}\right) \in$ $\mathbb{Z}^{8}$, defined as follows. $S L(2, \mathbb{Z})_{U}$ acts by left multiplication on the vectors:

$$
\left(\begin{array}{l}
Q_{1} \\
Q_{2}
\end{array}\right),\left(\begin{array}{c}
Q_{4} \\
-Q_{3}
\end{array}\right),\left(\begin{array}{l}
P_{1} \\
P_{2}
\end{array}\right),\left(\begin{array}{c}
P_{4} \\
-P_{3}
\end{array}\right) .
$$

This means, for example, that under an $S L(2, \mathbb{Z})_{U}$ transformation by a matrix $W$, we have $\left(\begin{array}{l}Q_{1}^{\prime} \\ Q_{2}^{\prime}\end{array}\right)=W\left(\begin{array}{l}Q_{1} \\ Q_{2}\end{array}\right)$. Similarly, $S L(2, \mathbb{Z})_{T}$ acts by left multiplication on the vectors:

$$
\left(\begin{array}{l}
Q_{1} \\
Q_{4}
\end{array}\right),\left(\begin{array}{c}
Q_{2} \\
-Q_{3}
\end{array}\right),\left(\begin{array}{l}
P_{1} \\
P_{4}
\end{array}\right),\left(\begin{array}{c}
P_{2} \\
-P_{3}
\end{array}\right)
$$

Finally $S L(2, \mathbb{Z})_{S}$ acts by left multiplication on the vectors:

$$
\left(\begin{array}{l}
Q_{1} \\
P_{1}
\end{array}\right),\left(\begin{array}{l}
Q_{2} \\
P_{2}
\end{array}\right),\left(\begin{array}{l}
Q_{3} \\
P_{3}
\end{array}\right),\left(\begin{array}{l}
Q_{4} \\
P_{4}
\end{array}\right) .
$$

In string theory, these transformation laws follow from the interpretation of $Q_{1}$ and $Q_{2}$ as the momentum quantum numbers along two circles that form part of the compact internal space, 


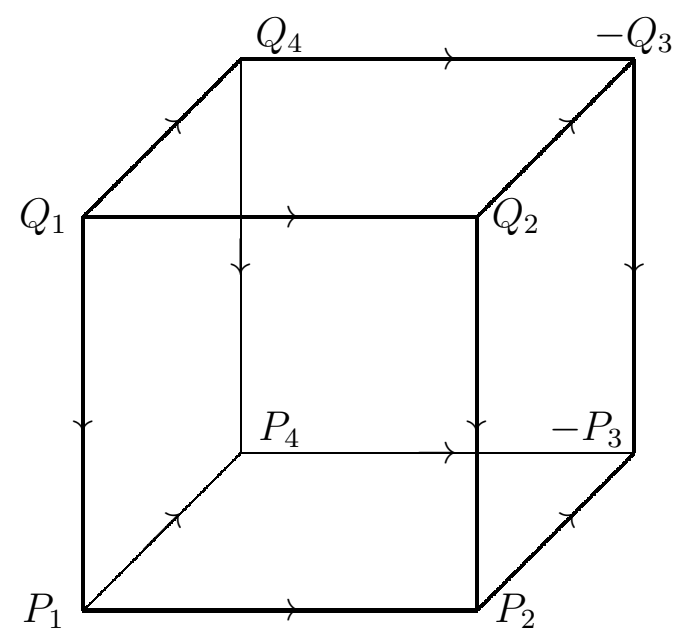

Figure 1: The Bhargava cube labelled by the eight dimensional dyonic charge. $\downarrow$ pairs charges that transform under $S L(2, \mathbb{Z})_{S}, \rightarrow$ pairs charges that transform under $S L(2, \mathbb{Z})_{U}$ and $\nearrow$ pairs charges that transform under $S L(2, \mathbb{Z})_{T}$.

$Q_{3}$ and $Q_{4}$ as the winding numbers along the same two circles and $P_{1}, \ldots, P_{4}$ as the magnetic counterparts of these charges.

The elements of the $\mathbb{Z}^{8}$ charge lattice can be grouped into equivalence classes - two elements are considered equivalent if there exists a duality transformation that maps one element to the other. We shall call these equivalence classes duality orbits. Our goal will be to classify the duality orbits of the theory.

Now given a pair of vectors $\left(\begin{array}{c}w_{1} \\ z_{1}\end{array}\right)$ and $\left(\begin{array}{c}w_{2} \\ z_{2}\end{array}\right)$ on which an $S L(2, \mathbb{Z})$ transformation acts by left multiplication, the combination $\left(w_{1} z_{2}-z_{1} w_{2}\right)$ is $S L(2, \mathbb{Z})$ invariant. Using this we get the following quadratic invariants of $S L(2, \mathbb{Z})_{U}, S L(2, \mathbb{Z})_{T}$ and $S L(2, \mathbb{Z})_{S}$ :

$$
\begin{aligned}
& S L(2, \mathbb{Z})_{U}: Q_{1} Q_{3}+Q_{2} Q_{4}, \quad P_{1} P_{3}+P_{2} P_{4}, \quad Q_{1} P_{2}-Q_{2} P_{1} \\
& Q_{3} P_{4}-Q_{4} P_{3}, \quad Q_{1} P_{3}+Q_{2} P_{4}, \quad Q_{4} P_{2}+Q_{3} P_{1}, \\
& S L(2, \mathbb{Z})_{T}: Q_{1} Q_{3}+Q_{2} Q_{4}, \quad P_{1} P_{3}+P_{2} P_{4}, \quad Q_{1} P_{4}-Q_{4} P_{1}, \\
& Q_{2} P_{3}-Q_{3} P_{2}, \quad Q_{1} P_{3}+Q_{4} P_{2}, \quad Q_{2} P_{4}+Q_{3} P_{1}, \\
& S L(2, \mathbb{Z})_{S} \quad: \quad Q_{1} P_{2}-Q_{2} P_{1}, \quad Q_{1} P_{3}-Q_{3} P_{1}, \quad Q_{1} P_{4}-Q_{4} P_{1}, \\
& Q_{2} P_{3}-Q_{3} P_{2}, \quad Q_{2} P_{4}-Q_{4} P_{2}, \quad Q_{3} P_{4}-Q_{4} P_{3} \text {. }
\end{aligned}
$$

Consider now a Bhargava cube with the entries at the eight corners as shown in Fig. 1. The cube has the property that a pair of entries connected by a top $\rightarrow$ down line transforms under 
$S L(2, \mathbb{Z})_{S}$, a pair of entries connected by a left $\rightarrow$ right line transforms under $S L(2, \mathbb{Z})_{U}$ and a pair of entries connected by a front $\rightarrow$ back line transforms under $S L(2, \mathbb{Z})_{T}$. We can now construct three binary quadratic forms associated with the three opposite pairs of faces of this cube. For a given pair of faces, the quadratic form is given by $-\operatorname{det}(M x+N y)$, where $M$ and $N$ are the integer matrices associated with the first and the second faces respectively. The top left corner of $M$ is always chosen to be the same integer, which we shall take to be $Q_{1}$. With this rule, the quadratic form associated with the top-down faces is

$$
\begin{aligned}
q_{1} & =-\operatorname{det}\left(\begin{array}{cc}
Q_{1} x+P_{1} y & Q_{2} x+P_{2} y \\
Q_{4} x+P_{4} y & -Q_{3} x-P_{3} y
\end{array}\right) \\
& =\left(Q_{1} Q_{3}+Q_{2} Q_{4}\right) x^{2}+\left(P_{1} P_{3}+P_{2} P_{4}\right) y^{2}+\left(Q_{1} P_{3}+Q_{3} P_{1}+Q_{2} P_{4}+Q_{4} P_{2}\right) x y .
\end{aligned}
$$

The coefficients are $S L(2, \mathbb{Z})_{U} \times S L(2, \mathbb{Z})_{T}$ invariant combinations. Similarly the quadratic form associated with the left-right faces takes the form:

$$
\begin{aligned}
q_{2} & =-\operatorname{det}\left(\begin{array}{ll}
Q_{1} x+Q_{2} y & P_{1} x+P_{2} y \\
Q_{4} x-Q_{3} y & P_{4} x-P_{3} y
\end{array}\right) \\
& =\left(Q_{4} P_{1}-Q_{1} P_{4}\right) x^{2}+\left(Q_{2} P_{3}-Q_{3} P_{2}\right) y^{2}+\left(Q_{4} P_{2}-Q_{2} P_{4}-Q_{3} P_{1}+Q_{1} P_{3}\right) x y,
\end{aligned}
$$

which has $S L(2, \mathbb{Z})_{S} \times S L(2, \mathbb{Z})_{T}$ invariant coefficients. Finally, the quadratic form associated with the front-back faces is given by

$$
\begin{aligned}
q_{3} & =-\operatorname{det}\left(\begin{array}{cc}
Q_{1} x+Q_{4} y & Q_{2} x-Q_{3} y \\
P_{1} x+P_{4} y & P_{2} x-P_{3} y
\end{array}\right) \\
& =\left(Q_{2} P_{1}-Q_{1} P_{2}\right) x^{2}+\left(Q_{4} P_{3}-Q_{3} P_{4}\right) y^{2}+\left(Q_{1} P_{3}-Q_{3} P_{1}-Q_{4} P_{2}+Q_{2} P_{4}\right) x y
\end{aligned}
$$

with $S L(2, \mathbb{Z})_{S} \times S L(2, \mathbb{Z})_{U}$ invariant coefficients.

Various duality transformations described in (2.1)-(2.3) by the $S L(2, \mathbb{Z})$ matrix $\left(\begin{array}{ll}\alpha & \beta \\ \gamma & \delta\end{array}\right)$ can be regarded as transformations of the variables $(x, y)$ of the form:

$$
(x, y) \mapsto\left(x^{\prime}, y^{\prime}\right)=(x, y)\left(\begin{array}{ll}
\alpha & \beta \\
\gamma & \delta
\end{array}\right) .
$$

For example the $S L(2, \mathbb{Z})_{S}$ transformation on $Q_{i}$ 's and $P_{i}$ 's described in (2.3) by the matrix $\left(\begin{array}{ll}\alpha & \beta \\ \gamma & \delta\end{array}\right)$ transforms the quadratic form (2.5) to a new one that can be obtained by making the replacement (2.8) in the original quadratic form. Similar remark holds for $S L(2, \mathbb{Z})_{U}$ and $S L(2, \mathbb{Z})_{T}$ transformations.

Given a quadratic form $A x^{2}+B x y+C y^{2}$, we have defined the discriminant $D$ to be $B^{2}-$ $4 A C$. It is easy to verify that all of the quadratic forms (2.5), (2.6) and (2.7) have the same 
discriminant $D$ given by the $S L(2, \mathbb{Z})_{S} \times S L(2, \mathbb{Z})_{T} \times S L(2, \mathbb{Z})_{U}$ invariant quartic combination of the charges:

$$
D=-4\left(Q_{1} Q_{3}+Q_{2} Q_{4}\right)\left(P_{1} P_{3}+P_{2} P_{4}\right)+\left(Q_{1} P_{3}+Q_{3} P_{1}+Q_{2} P_{4}+Q_{4} P_{2}\right)^{2} .
$$

We now turn to the problem of classification of the duality orbits of $S L(2, \mathbb{Z})_{S} \times S L(2, \mathbb{Z})_{T} \times$ $S L(2, \mathbb{Z})_{U}$. In this section, we shall consider the case where each of the quadratic forms given in (2.5), (2.6) and (2.7) is primitive, i.e. $A, B, C$ are coprime for each of them. It can be easily argued that this condition is invariant under the duality group, i.e. starting with a cube where all three quadratic forms are primitive, we cannot make a duality transformation that makes one or more of them non-primitive. In this case, to each of the quadratic forms we can associate an element of the class group for discriminant $D$, consisting of the equivalence classes of binary quadratic forms up to $S L(2, \mathbb{Z})$ transformations. Naively, there will be three such elements that will characterize a given duality orbit. However, since the three class group elements are associated with three opposite pairs of faces of a Bhargava cube, the composition of the three elements gives the identity element of the group by Bhargava's theorem [12]. Therefore only two of them are independent. It also follows from Bhargava's result that two different charge vectors, for which the corresponding Bhargava cubes have the same (primitive) class group elements, can be related to each other by duality transformation. The proof of these results have been reviewed in Appendix C. Therefore we can use any two of the three class group elements associated with the quadratic forms (2.5), (2.6) and (2.7) to characterize a duality orbit of $S L(2, \mathbb{Z})_{S} \times S L(2, \mathbb{Z})_{T} \times S L(2, \mathbb{Z})_{U}$. For definiteness we shall choose the class group elements associated with the quadratic forms (2.5) and (2.6) to label the duality orbit. As is well known [11,27, 28], these class group elements can be represented uniquely by quadratic forms of the type $A x^{2}+B x y+C y^{2}$ with $A, B, C$ satisfying the following conditions:

$$
|B| \leq|A| \leq|C|, \quad A B>0 \quad \text { for } \quad|B|=|A| \quad \text { or } \quad A=C
$$

\section{$3 \quad S L(2, \mathbb{Z})^{3}$ orbits: Non-primitive case}

Only primitive quadratic forms $A x^{2}+B x y+C y^{2}$ form elements of class groups obeying group composition laws. However even when the quadratic form is not primitive, one can still define the notion of equivalence classes under $S L(2, \mathbb{Z})$ transformations, e.g. different quadratic forms (2.5) related by $S L(2, \mathbb{Z})_{S}$ transformations are considered equivalent. Furthermore, the inequivalent quadratic forms are still characterized by restricting the coefficients to satisfy 
(2.10), but now we do not demand $A, B$ and $C$ to be coprime. Therefore a natural question would be: even when the quadratic forms (2.5) and / or (2.6) are not primitive, do their equivalence classes under $S L(2, \mathbb{Z})_{S}$ and $S L(2, \mathbb{Z})_{U}$ transformations determine the duality orbit uniquely?

We begin by introducing some notations. Let us denote the quadratic forms (2.5) and (2.6) by:

$$
\begin{aligned}
q_{1} & \equiv A_{1} x^{2}+B_{1} x y+C_{1} y^{2} \\
& =\left(Q_{1} Q_{3}+Q_{2} Q_{4}\right) x^{2}+\left(P_{1} P_{3}+P_{2} P_{4}\right) y^{2}+\left(Q_{1} P_{3}+Q_{3} P_{1}+Q_{2} P_{4}+Q_{4} P_{2}\right) x y \\
q_{2} & \equiv A_{2} x^{2}+B_{2} x y+C_{2} y^{2} \\
& =\left(Q_{4} P_{1}-Q_{1} P_{4}\right) x^{2}+\left(Q_{2} P_{3}-Q_{3} P_{2}\right) y^{2}+\left(Q_{4} P_{2}-Q_{2} P_{4}-Q_{3} P_{1}+Q_{1} P_{3}\right) x y
\end{aligned}
$$

We also define the minor matrix for the Bhargava cube $\mathcal{A}$ depicted in Fig. 1 as

$$
M(\mathcal{A})=\left(\begin{array}{cccc}
Q_{1} & Q_{2} & P_{1} & P_{2} \\
Q_{4} & -Q_{3} & P_{4} & -P_{3}
\end{array}\right)
$$

Clearly, the minor matrix carries exactly the same information as the Bhargava cube, but the information is represented in an asymmetric manner. It can be easily checked that

$$
\begin{aligned}
& M_{12}=-A_{1}, \quad M_{13}=-A_{2}, \quad M_{14}=-\frac{B_{2}+B_{1}}{2}, \\
& M_{34}=-C_{1}, \quad M_{24}=-C_{2}, \quad M_{23}=-\frac{B_{2}-B_{1}}{2},
\end{aligned}
$$

where $M_{i j}$ denotes the minor of the matrix $M(\mathcal{A})$, obtained by keeping only the $i$ 'th and $j$ 'th column of $M(\mathcal{A})$ and then taking the determinant of the corresponding square matrix. Note that $\left(B_{1} \pm B_{2}\right) / 2$ are integers since $q_{1}$ and $q_{2}$ have the same discriminants.

Now we record a Lemma due to Gauss which we need to carry out our analysis.

Lemma 3.1. (Gauss's Lemma) Let

$$
M=\left(\begin{array}{llll}
p_{1} & p_{2} & \ldots & p_{n} \\
r_{1} & r_{2} & \ldots & r_{n}
\end{array}\right) \quad \text { and } \quad M^{\prime}=\left(\begin{array}{cccc}
p_{1}^{\prime} & p_{2}^{\prime} & \ldots & p_{n}^{\prime} \\
r_{1}^{\prime} & r_{2}^{\prime} & \ldots & r_{n}^{\prime}
\end{array}\right)
$$

be two $2 \times n$-matrices $(n \geq 3)$ with the following properties:

1. the $2 \times 2-$ minors of $M$ are coprime;

2. there is an integer $m$ such that each minor of $M^{\prime}$ is $m$ times the corresponding minor of $M$. 
Then there is a matrix $g=\left(\begin{array}{ll}a & b \\ c & d\end{array}\right)$ with determinant $m$ and $a, b, c, d \in \mathbb{Z}$, such that $M^{\prime}=g M$.

The proof of this Lemma has been reviewed in Appendix A.

We now return to our problem. Suppose that the quadratic forms $q_{1}$ and $q_{2}$ given in (3.1) are not primitive, but that $\left(A_{1}, B_{1}, C_{1}, A_{2}, B_{2}, C_{2}\right)$ are coprime. This implies that $\left(A_{1}, C_{1}, A_{2}, C_{2},\left(B_{1}+\right.\right.$ $\left.\left.B_{2}\right) / 2,\left(B_{1}-B_{2}\right) / 2\right)$ are also coprime. Now suppose that we have another set of charges $\left(Q_{1}^{\prime}, \ldots, Q_{4}^{\prime}, P_{1}^{\prime}, \ldots, P_{4}^{\prime}\right)$ with the same quadratic forms $q_{1}$ and $q_{2}$ given in (3.1). Then by Gauss's Lemma the corresponding minor matrix

$$
M\left(\mathcal{A}^{\prime}\right)=\left(\begin{array}{cccc}
Q_{1}^{\prime} & Q_{2}^{\prime} & P_{1}^{\prime} & P_{2}^{\prime} \\
Q_{4}^{\prime} & -Q_{3}^{\prime} & P_{4}^{\prime} & -P_{3}^{\prime}
\end{array}\right)
$$

must be related to $M(\mathcal{A})$ defined in $(3.2)$ by an $S L(2, \mathbb{Z})$ transformation:

$$
M\left(\mathcal{A}^{\prime}\right)=\left(\begin{array}{ll}
\alpha & \beta \\
\gamma & \delta
\end{array}\right) M(\mathcal{A}), \quad \alpha, \beta, \gamma, \delta \in \mathbb{Z}, \quad \alpha \delta-\beta \gamma=1
$$

Using (2.2) we can identify this as an $S L(2, \mathbb{Z})_{T}$ transformation. Therefore the charge vectors $\left(Q_{1}, \ldots, Q_{4}, P_{1}, \ldots, P_{4}\right)$ and $\left(Q_{1}^{\prime}, \ldots, Q_{4}^{\prime}, P_{1}^{\prime}, \ldots, P_{4}^{\prime}\right)$ are in the same duality orbit.

If we consider the more general case where the quadratic forms $q_{1}^{\prime}$ and $q_{2}^{\prime}$ associated with the charge vector $\left(Q_{1}^{\prime}, \ldots, Q_{4}^{\prime}, P_{1}^{\prime}, \ldots, P_{4}^{\prime}\right)$ are not the same as $q_{1}$ and $q_{2}$ but are in the same equivalence classes, then we can first apply an $S L(2, \mathbb{Z})_{S}$ transformation to make $q_{1}^{\prime}=q_{1}$, and then apply an $S L(2, \mathbb{Z})_{U}$ transformation to make $q_{2}^{\prime}=q_{2}$. Then we can use Gauss's Lemma to show that the two charge vectors are related by $S L(2, \mathbb{Z})_{T}$ transformation.

Therefore we conclude that if $\left(A_{1}, B_{1}, C_{1}, A_{2}, B_{2}, C_{2}\right)$ are coprime, then the equivalence classes of the quadratic forms given in (3.1) uniquely determine the duality orbit. Put another way, two charge vectors, for which the equivalence classes of the quadratic forms given in (3.1) agree, are related by a duality transformation.

In order to complete the analysis, we need to check that given a pair of quadratic forms of the form shown in (3.1), there exists a Bhargava cube that generates these quadratic forms. This has been proved in Appendix B following the same method that is used to prove the existence of Bhargava cube for a pair of primitive quadratic forms.

Finally, consider the case where $\left(A_{1}, B_{1}, C_{1}, A_{2}, B_{2}, C_{2}\right)$ are not coprime but have some common factor $m>1$ :

$$
m=\operatorname{gcd}\left(A_{1}, B_{1}, C_{1}, A_{2}, B_{2}, C_{2}\right)
$$


In that case, we can define another pair of quadratic forms:

$$
\widetilde{A}_{1} x^{2}+\widetilde{B}_{1} x y+\widetilde{C}_{1} y^{2}=\frac{1}{m}\left(A_{1} x^{2}+B_{1} x y+C_{1} y^{2}\right), \quad \widetilde{A}_{2} x^{2}+\widetilde{B}_{2} x y+\widetilde{C}_{2} y^{2}=\frac{1}{m}\left(A_{2} x^{2}+B_{2} x y+C_{2} y^{2}\right),
$$

so that $\left(\widetilde{A}_{1}, \widetilde{B}_{1}, \widetilde{C}_{1}, \widetilde{A}_{2}, \widetilde{B}_{2}, \widetilde{C}_{2}\right)$ are coprime. Let $M_{0}$ be a minor matrix that represents this new pair of quadratic forms via the analog of (3.3). Of course, $M_{0}$ is only defined up to an $S L(2, \mathbb{Z})_{T}$ transformation, but we choose one of these matrices. It now follows from Gauss's Lemma that the original minor matrix $M(\mathcal{A})$ defined in $(3.2)$ is given by

$$
M(\mathcal{A})=g M_{0}
$$

where $g$ is an integer matrix with determinant $m$.

Now let us consider another set of charges $\left(Q_{1}^{\prime}, \ldots, Q_{4}^{\prime}, P_{1}^{\prime}, \ldots, P_{4}^{\prime}\right)$ for which the associated quadratic forms are the same as $\left(q_{1}, q_{2}\right) 4$ Gauss's Lemma tells us that the minor matrix $M\left(\mathcal{A}^{\prime}\right)$ associated with the new charges is given by

$$
M\left(\mathcal{A}^{\prime}\right)=g^{\prime} M_{0},
$$

where $g^{\prime}$ is another integer matrix with determinant $m$.

Now the two sets of charge vectors are in the same duality orbit if $M(\mathcal{A})$ and $M\left(\mathcal{A}^{\prime}\right)$ are related by multiplication by an $S L(2, \mathbb{Z})$ transformation from the left. Therefore we need to determine whether $g$ and $g^{\prime}$ are related by an $S L(2, \mathbb{Z})$ transformation from the left. We shall prove below that any $2 \times 2$ matrix $g$ with integer entries and determinant $m$ can be written as,

$$
g=S U, \quad U=\left(\begin{array}{ll}
a & b \\
0 & d
\end{array}\right), \quad S \in S L(2, \mathbb{Z}), \quad a d=m, \quad a, d>0, \quad 0 \leq b \leq d-1 .
$$

Furthermore the decomposition is unique, i.e. for any given matrix $g$, the matrices $S$ and $U$ are determined without any ambiguity. Therefore the inequivalent duality orbits will be classified by different values of $a, b, d$, besides the equivalence classes of the quadratic forms given in (2.5) and (2.6). The decomposition (3.11) generalizes a construction of [21] for prime values of $m$.

We now give a proof of the decomposition (3.11) and its uniqueness. Since (3.11) gives $\operatorname{det} g=m$, it follows from (3.7), that $\operatorname{det} g>1$. We will explicitly construct the matrices $U$

\footnotetext{
${ }^{4}$ As before, if the quadratic classes associated with the charges $\left(Q_{1}^{\prime}, \ldots, Q_{4}^{\prime}, P_{1}^{\prime}, \ldots, P_{4}^{\prime}\right)$ are not the same as $\left(q_{1}, q_{2}\right)$ but are in the same equivalence classes, we can make them same by appropriate $S L(2, \mathbb{Z})_{S} \times S L(2, \mathbb{Z})_{U}$ transformations.
} 
and $S$ satisfying (3.11). Let

$$
g=\left(\begin{array}{ll}
r & s \\
t & u
\end{array}\right), \quad U=\left(\begin{array}{ll}
a & b \\
0 & d
\end{array}\right) \quad \text { and } \quad S=\left(\begin{array}{ll}
\alpha & \beta \\
\gamma & \delta
\end{array}\right)
$$

We will find $a, b, d, \alpha, \beta, \gamma$ and $\delta$ in terms of the entries of $g$. Observe that $U=S^{-1} g$ gives

$$
\left(\begin{array}{ll}
a & b \\
0 & d
\end{array}\right)=\left(\begin{array}{cc}
\delta & -\beta \\
-\gamma & \alpha
\end{array}\right)\left(\begin{array}{ll}
r & s \\
t & u
\end{array}\right)=\left(\begin{array}{cc}
\delta r-\beta t & \delta s-\beta u \\
-\gamma r+\alpha t & -\gamma s+\alpha u
\end{array}\right)
$$

Thus we must have $\gamma r=\alpha t$. We choose $\gamma=t / \operatorname{gcd}(r, t)$ and $\alpha=r / \operatorname{gcd}(r, t)$. Since this gives $\operatorname{gcd}(\alpha, \gamma)=1$, by Euclid's algorithm, we can find integers $\beta$ and $\delta$ such that $\alpha \delta-\beta \gamma=1$. Thus $S$ constructed in this manner has determinant 1 . The integers $a, b$ and $d$ can now be read out from the right hand side of (3.13). Taking determinant of both sides of the equation $g=S U$, we get that $m=a d$. Since $a d$ is positive, we can make $a$ and $d$ positive by multiplying $U$ and $S$ by $-I_{2}$ if necessary. Here $I_{2}$ denotes the $2 \times 2$ identity matrix. To get $0 \leq b \leq d-1$, suppose $b>d$ or $b<0$. Then $b=h d+\widetilde{b}$ where $0 \leq \widetilde{b} \leq d-1$ and $h \in \mathbb{Z}, h \neq 0$. We then have

$$
\left(\begin{array}{ll}
a & b \\
0 & d
\end{array}\right)=\left(\begin{array}{ll}
1 & h \\
0 & 1
\end{array}\right)\left(\begin{array}{ll}
a & \widetilde{b} \\
0 & d
\end{array}\right)
$$

Thus we have

$$
\left(\begin{array}{ll}
r & s \\
t & u
\end{array}\right)=\left(\begin{array}{ll}
\alpha & \beta \\
\gamma & \delta
\end{array}\right)\left(\begin{array}{ll}
1 & h \\
0 & 1
\end{array}\right)\left(\begin{array}{ll}
a & \widetilde{b} \\
0 & d
\end{array}\right)
$$

So rename $S=\left(\begin{array}{ll}\alpha & \beta \\ \gamma & \delta\end{array}\right)\left(\begin{array}{ll}1 & h \\ 0 & 1\end{array}\right)$ and $U=\left(\begin{array}{ll}a & \widetilde{b} \\ 0 & d\end{array}\right)$. This establishes the existence of $U$ and $S$ satisfying (3.11).

The analysis above has not yet established that for given $r, s, t, u$, the choice of $S$ and $U$ is unique. To prove uniqueness, suppose that $g=S^{\prime} U^{\prime}$ where

$$
U^{\prime}=\left(\begin{array}{ll}
a^{\prime} & b^{\prime} \\
0 & d^{\prime}
\end{array}\right) \quad \text { and } \quad S^{\prime}=\left(\begin{array}{cc}
\alpha^{\prime} & \beta^{\prime} \\
\gamma^{\prime} & \delta^{\prime}
\end{array}\right) \in S L(2, \mathbb{Z}), \quad a^{\prime}, d^{\prime}>0, \quad 0 \leq b^{\prime} \leq d^{\prime}-1 .
$$

Then $S U=S^{\prime} U^{\prime}$ tells us that $S^{-1} S^{\prime} U^{\prime}=U$. Using the form of $U, U^{\prime}$, it follows immediately that the lower left corner of $S^{-1} S^{\prime}$ vanishes. Therefore, $S^{-1} S^{\prime}$ must have the form $\left(\begin{array}{ll}1 & k \\ 0 & 1\end{array}\right)$ for some $k \in \mathbb{Z}$. This gives $a^{\prime}=a, d^{\prime}=d, b^{\prime}=b-k d$. However since $0 \leq b, b^{\prime} \leq d-1, k$ must vanish and we have $b^{\prime}=b$. This shows that $S^{\prime}=S$ and $U^{\prime}=U$.

We still need to check that the different choices of $a, b, d$ in (3.11) always produce inequivalent $M(\mathcal{A})$, i.e. there are no special matrices of the form $M_{0}$ for which

$$
U M_{0}=S U^{\prime} M_{0}, \quad U^{\prime} \equiv\left(\begin{array}{cc}
a^{\prime} & b^{\prime} \\
0 & d^{\prime}
\end{array}\right)
$$


for some $S L(2, \mathbb{Z})$ matrix $S$. Since the quadratic forms associated with the minor matrix are not identically zero, it follows from (3.3) that at least one matrix $\mathcal{M}_{i j}$, obtained by keeping the $i$-th and the $j$-th columns of $M_{0}$, must have non-vanishing determinant. Now, it follows from (3.17) that

$$
U \mathcal{M}_{i j}=S U^{\prime} \mathcal{M}_{i j}
$$

Since $\mathcal{M}_{i j}$ has an inverse, we get $U=S U^{\prime}$. However, due to the uniqueness of the decomposition given in (3.11), this is possible only for $U=U^{\prime}$ and $S=I_{2}$ as long as $a, b, d$ and $a^{\prime}, b^{\prime}, d^{\prime}$ lie in the range given in (3.11). Therefore different choices of $(a, b, d)$ in (3.11) produce inequivalent $M(\mathcal{A})$.

There are some special quadratic forms for which we need to be more careful. Let us suppose that the quadratic form (2.5) and/or (2.6) is such that it is invariant under some $S L(2, \mathbb{Z})_{S}$ and/or $S L(2, \mathbb{Z})_{U}$ transformation [21]. For example the quadratic form $x^{2}+y^{2}$ is invariant under $\left(\begin{array}{cc}0 & -1 \\ 1 & 0\end{array}\right)$. In that case two different charge vectors, related by this $S L(2, \mathbb{Z})_{S}$ and/or $S L(2, \mathbb{Z})_{U}$ transformation, will give same expressions for (2.5) and (2.6). Therefore in order to determine if $U$ and $U^{\prime}$ describe the same duality orbit, we could generalize (3.17) by allowing this $S L(2, \mathbb{Z})_{S}$ and/or $S L(2, \mathbb{Z})_{U}$ transformation to act on the right hand side. Now from the duality transformation laws (2.1) $-(2.3)$ and the expression for the matrix $M(\mathcal{A})$ given in $(3.2)$, we see that an $S L(2, \mathbb{Z})_{S} \times S L(2, \mathbb{Z})_{U}$ transformation can be represented by a right multiplication of $M(\mathcal{A})$ by some $4 \times 4$ matrix $V 5$ Therefore in order that $(a, b, d)$ and $\left(a^{\prime}, b^{\prime}, d^{\prime}\right)$ produce equivalent $M(\mathcal{A})$, it is enough to demand that

$$
U M_{0}=S U^{\prime} M_{0} V
$$

where $V$ represents some $S L(2, \mathbb{Z})_{S} \times S L(2, \mathbb{Z})_{U}$ transformation that leaves the quadratic forms (2.5) and (2.6) unchanged. Now since $M_{0} V$ and $M_{0}$ generate the same quadratic forms, by Gauss's Lemma we have

$$
M_{0} V=\widetilde{S}\left(M_{0}, V\right) M_{0}
$$

for some $S L(2, \mathbb{Z})$ matrix $\widetilde{S}\left(M_{0}, V\right)$ that depends on both $M_{0}$ and $V$. Substituting this into the right hand side of (3.19), we get

$$
U=S U^{\prime} \widetilde{S}\left(M_{0}, V\right)
$$

where we have used arguments similar to the one given below (3.18) to remove $M_{0}$ from both sides.

\footnotetext{
${ }^{5} \mathrm{~V}$ is actually an $S O(2,2 ; \mathbb{Z})$ matrix, but this will not be important for our analysis.
} 
As reviewed in Appendix D, the only special quadratic forms that are invariant under some $S L(2, \mathbb{Z})$ transformation other than $\pm I_{2}$ are those proportional to $x^{2}+y^{2}$ or $x^{2}+x y+y^{2}[21,22$ ] 6 , It has been shown in Appendix E that in all cases, by suitably choosing $M_{0}$, the matrix $\widetilde{S}\left(M_{0}, V\right)$ can be brought to one of the following three forms:

$$
\left(\begin{array}{cc}
0 & -1 \\
1 & 0
\end{array}\right), \quad\left(\begin{array}{cc}
1 & -1 \\
1 & 0
\end{array}\right), \quad\left(\begin{array}{cc}
0 & -1 \\
1 & -1
\end{array}\right)
$$

The first one is relevant when $q_{1}$ and/or $q_{2}$ is proportional to $\left(x^{2}+y^{2}\right)$, while the second and the third ones are relevant when $q_{1}$ and/or $q_{2}$ is proportional to $\left(x^{2}+x y+y^{2}\right)$.

Let us now try to find solutions to (3.21). For $\widetilde{S}\left(M_{0}, V\right)=\left(\begin{array}{cc}0 & -1 \\ 1 & 0\end{array}\right)$, the equation takes the form:

$$
\left(\begin{array}{ll}
a & b \\
0 & d
\end{array}\right)=\left(\begin{array}{ll}
\alpha & \beta \\
\gamma & \delta
\end{array}\right)\left(\begin{array}{ll}
a^{\prime} & b^{\prime} \\
0 & d^{\prime}
\end{array}\right)\left(\begin{array}{cc}
0 & -1 \\
1 & 0
\end{array}\right), \quad\left(\begin{array}{ll}
\alpha & \beta \\
\gamma & \delta
\end{array}\right) \in S L(2, \mathbb{Z}) .
$$

This equation can be rewritten as

$$
\left(\begin{array}{ll}
a^{\prime} & b^{\prime} \\
0 & d^{\prime}
\end{array}\right)=\left(\begin{array}{cc}
-\delta b+\beta d & \delta a \\
\gamma b-\alpha d & -\gamma a
\end{array}\right)
$$

Comparing the lower left corner, we get $\gamma b=\alpha d$. Since $\gamma$ and $\alpha$ are coprime, this can be solved by taking $\gamma=-d / \operatorname{gcd}(b, d), \alpha=-b / \operatorname{gcd}(b, d)$. We can now find $\delta$ and $\beta$ satisfying $\alpha \delta-\beta \gamma=1$, but the result is determined only up to a shift symmetry: $\delta \rightarrow \delta+r \gamma, \beta \rightarrow \beta+r \alpha$, $r \in \mathbb{Z}$. Comparing the lower right corners we now get $d^{\prime}=-\gamma a=a d / \operatorname{gcd}(b, d)$. Comparing the determinants on both sides we get $a^{\prime}=\operatorname{gcd}(b, d)$. Comparison of the upper left corners gives $-\delta b+\beta d=\operatorname{gcd}(b, d)$, but this is the same as the equation $\alpha \delta-\beta \gamma=1$. Comparison of the upper right corner gives $b^{\prime}=\delta a$. Due to the shift symmetry mentioned above, $b^{\prime}$ is determined up to an additive factor of $r \gamma a=-r d^{\prime}, r \in \mathbb{Z}$. Using this freedom, we can bring $b^{\prime}$ in the range $0 \leq b^{\prime} \leq d^{\prime}-1$. Therefore the final results for $a^{\prime}, b^{\prime}, d^{\prime}$ may be written as,

$$
a^{\prime}=\operatorname{gcd}(b, d), \quad d^{\prime}=a d / \operatorname{gcd}(b, d), \quad b^{\prime} \equiv \delta a \bmod d^{\prime}, 0 \leq b^{\prime}<d^{\prime}
$$

where $(\delta, \beta)$ are any integer solutions to the equation

$$
-\delta b+\beta d=\operatorname{gcd}(b, d) .
$$

\footnotetext{
${ }^{6}$ The $S L(2, \mathbb{Z})$ transformation $-I_{2}$ leaves every quadratic form unchanged, but since the corresponding $\widetilde{S}\left(M_{0}, V\right)$ is $-I_{2}$, it can be absorbed into the definition of $S$ in (3.21) and does not affect $U^{\prime}$. We need to be a bit more careful in the next section where $\Gamma_{T}$ represents a congruence subgroup of $S L(2, \mathbb{Z})$. If this subgroup does not include $-I_{2}$, then we cannot absorb $-I_{2}$ into the definition of $S$ in (3.21). See footnote 8 for a discussion on this.
} 
This tells us that when $q_{1}$ and/or $q_{2}$ is proportional to $x^{2}+y^{2}$, the orbits labelled by $(a, b, d)$ and $\left(a^{\prime}, b^{\prime}, d^{\prime}\right)$ are the same if $\left(a^{\prime}, b^{\prime}, d^{\prime}\right)$ are related to $(a, b, d)$ via (3.25), (3.26).

For $\widetilde{S}\left(M_{0}, V\right)=\left(\begin{array}{cc}1 & -1 \\ 1 & 0\end{array}\right)$, the equation takes the form:

$$
\left(\begin{array}{ll}
a & b \\
0 & d
\end{array}\right)=\left(\begin{array}{ll}
\alpha & \beta \\
\gamma & \delta
\end{array}\right)\left(\begin{array}{ll}
a^{\prime} & b^{\prime} \\
0 & d^{\prime}
\end{array}\right)\left(\begin{array}{cc}
1 & -1 \\
1 & 0
\end{array}\right)
$$

Equation (3.27) can be written as:

$$
\left(\begin{array}{ll}
a^{\prime} & b^{\prime} \\
0 & d^{\prime}
\end{array}\right)=\left(\begin{array}{cc}
-\delta b+\beta d & \delta(a+b)-\beta d \\
\gamma b-\alpha d & -\gamma(a+b)+\alpha d
\end{array}\right)
$$

This equation can be analyzed in the same way as (3.24) leading to the result:

$$
a^{\prime}=\operatorname{gcd}(b, d), \quad d^{\prime}=\frac{a d}{\operatorname{gcd}(b, d)}, \quad b^{\prime}=a^{\prime}+\delta a, \quad \beta d-\delta b=\operatorname{gcd}(b, d) .
$$

The solution $(\beta, \delta)$ of the last equation has to be chosen so that $b^{\prime}$ lies in the range $\left(0, \ldots, d^{\prime}-1\right)$.

For $\widetilde{S}\left(M_{0}, V\right)=\left(\begin{array}{ll}0 & -1 \\ 1 & -1\end{array}\right)$, the equation takes the form:

$$
\left(\begin{array}{ll}
a & b \\
0 & d
\end{array}\right)=\left(\begin{array}{ll}
\alpha & \beta \\
\gamma & \delta
\end{array}\right)\left(\begin{array}{ll}
a^{\prime} & b^{\prime} \\
0 & d^{\prime}
\end{array}\right)\left(\begin{array}{ll}
0 & -1 \\
1 & -1
\end{array}\right)
$$

We can write this as

$$
\left(\begin{array}{ll}
a^{\prime} & b^{\prime} \\
0 & d^{\prime}
\end{array}\right)=\left(\begin{array}{cc}
-\delta(a+b)+\beta d & \delta a \\
\gamma(a+b)-\alpha d & -\gamma a
\end{array}\right)
$$

This equation can also be analyzed in the same way as (3.24) leading to the result:

$$
a^{\prime}=\operatorname{gcd}(a+b, d), \quad d^{\prime}=\frac{a d}{\operatorname{gcd}(a+b, d)}, \quad b^{\prime}=\delta a, \quad \beta d-\delta(a+b)=\operatorname{gcd}(a+b, d) .
$$

Again, the solution $(\beta, \delta)$ of the last equation has to be chosen so that $b^{\prime}$ lies in the range $\left(0, \ldots, d^{\prime}-1\right)$.

Therefore, when $q_{1}$ and/or $q_{2}$ is proportional to $x^{2}+x y+y^{2}$, the orbits labelled by $(a, b, d)$ and $\left(a^{\prime}, b^{\prime}, d^{\prime}\right)$ are the same if $\left(a^{\prime}, b^{\prime}, d^{\prime}\right)$ are related to $(a, b, d)$ via (3.29) or (3.32).

\section{Orbits of products of congruence subgroups of $S L(2, \mathbb{Z})$}

Let us now suppose that the actual duality group is $\Gamma_{S} \times \Gamma_{T} \times \Gamma_{U}$ where $\Gamma_{S}, \Gamma_{T}$ and $\Gamma_{U}$ are congruence subgroups of $S L(2, \mathbb{Z})$. We shall allow them to be distinct congruence subgroups. Our goal will be to classify the duality orbits of this theory. 
The first task will be to classify the equivalence classes of binary quadratic forms under the action of a given congruence subgroup $\Gamma$ of $S L(2, \mathbb{Z})$. This problem was studied in [23]. Given a quadratic form $A x^{2}+B x y+C y^{2}$, we consider the solution to the equation: 7

$$
C \tau^{2}+B \tau+A=0
$$

For $B^{2}<4 A C$, this equation has a unique solution lying in the upper half plane:

$$
\tau=\left\{-\frac{B}{2 C}+\frac{i}{2|C|} \sqrt{4 A C-B^{2}}\right\} .
$$

We now choose a fundamental domain of $\Gamma$ in the upper half plane, and choose $A, B, C$ in the range so that $\tau$ lies inside the fundamental domain. For $\Gamma=S L(2, \mathbb{Z})$ this produces the range (2.10). For $\Gamma_{0}(p)$ with $p=2,3$, the restriction on $A, B, C$ may be chosen to be [23]:

$$
|B| \leq|C|, \quad|B| \leq p|A|, \quad A B>0 \quad \text { for } \quad|B|=|C| \quad \text { or } \quad|B|=p|A| .
$$

Similar results can be derived for other congruence subgroups of $S L(2, \mathbb{Z})$. It follows from the analysis of [1 3] that $\tau$ given in (4.2) for the quadratic forms (2.5), (2.6), (2.7) give the attractor values of the $S, U$ and $T$ moduli respectively of supersymmetric black holes of charge $\left(Q_{1}, \ldots, Q_{4}, P_{1}, \ldots, P_{4}\right)$, but this will not play any role in our analysis.

Now suppose that we are given a charge vector and the associated Bhargava cube. For this we can determine the $\Gamma_{S}$ and $\Gamma_{U}$ equivalence classes of (2.5) and (2.6), by making appropriate $\Gamma_{S}$ and $\Gamma_{U}$ transformations to bring the quadratic forms in the chosen range of values of $A, B, C$. This can be done irrespective of whether the quadratic forms are primitive or not. The question that we need to address now is: given the $\Gamma_{S}$ and $\Gamma_{U}$ equivalence classes of (2.5) and (2.6), what are the possible duality orbits?

Now, by following the analysis of $\S 3$ we can show that the matrix $M(\mathcal{A})$ constructed in (3.2) can be expressed as in (3.9) for some reference matrix $M_{0}$ and an integer matrix $g$ of determinant $m$, with $m$ defined as in (3.7). Since $\Gamma_{T}$ acts on $M(\mathcal{A})$ by left multiplication, we see that different $g$, related by left multiplication by elements of $\Gamma_{T}$, produce charge vectors in the same duality orbit. Therefore we can classify the independent duality orbits as $\Gamma_{T} \backslash G$, where $G$ is the set of integer matrices of determinant $m$. The solution to this problem may be found as follows. We have seen in (3.11) that any integer matrix of determinant $m$ can be expressed as the product of an $S L(2, \mathbb{Z})$ matrix $S$ and a matrix $U$ of the form given in

\footnotetext{
${ }^{7}$ Note that due to the transformation law (2.8) of $x, y$ under $\Gamma$, our variables $x$ and $y$ are related to those in 23 by $x \leftrightarrow y$ exchange.
} 
(3.11). On the other hand, any $S L(2, \mathbb{Z})$ matrix can be expressed as an element of $\Gamma_{T}$ and a representative element of the coset $\Gamma_{T} \backslash S L(2, \mathbb{Z})$. Therefore the matrix $g$ can be expressed as

$$
S^{\prime} \mathcal{G} U, \quad S^{\prime} \in \Gamma_{T}, \quad \mathcal{G} \in \Gamma_{T} \backslash S L(2, \mathbb{Z}), \quad U=\left(\begin{array}{ll}
a & b \\
0 & d
\end{array}\right),
$$

In that case the different possible choices of $\Gamma_{T}$ inequivalent $g$ appearing in the decomposition (3.9) may be taken to be

$$
\mathcal{G}\left(\begin{array}{ll}
a & b \\
0 & d
\end{array}\right), \quad \mathcal{G} \in \Gamma_{T} \backslash S L(2, \mathbb{Z})
$$

In order to check that these always generate inequivalent duality orbits, we also need to check that we cannot have,

$$
\mathcal{G}\left(\begin{array}{ll}
a & b \\
0 & d
\end{array}\right) M_{0}=S^{\prime} \mathcal{G}^{\prime}\left(\begin{array}{ll}
a^{\prime} & b^{\prime} \\
0 & d^{\prime}
\end{array}\right) M_{0}, \quad S^{\prime} \in \Gamma_{T}, \quad \mathcal{G}, \mathcal{G}^{\prime} \in \Gamma_{T} \backslash S L(2, \mathbb{Z}),
$$

unless $S^{\prime}$ is identify, $\mathcal{G}^{\prime}=\mathcal{G}$ and $\left(\begin{array}{ll}a & b \\ 0 & d\end{array}\right)=\left(\begin{array}{ll}a^{\prime} & b^{\prime} \\ 0 & d^{\prime}\end{array}\right)$. To prove this we express this equation as,

$$
\left(\begin{array}{ll}
a & b \\
0 & d
\end{array}\right) M_{0}=\mathcal{G}^{-1} S^{\prime} \mathcal{G}^{\prime}\left(\begin{array}{ll}
a^{\prime} & b^{\prime} \\
0 & d^{\prime}
\end{array}\right) M_{0}
$$

Since $\mathcal{G}^{-1} S^{\prime} \mathcal{G} \in S L(2, \mathbb{Z})$, it follows from the analysis below (3.17) that we must have $\left(\begin{array}{ll}a & b \\ 0 & d\end{array}\right)=$ $\left(\begin{array}{cc}a^{\prime} & b^{\prime} \\ 0 & d^{\prime}\end{array}\right)$ and $\mathcal{G}^{-1} S^{\prime} \mathcal{G}^{\prime}$ must be identity. Therefore $\mathcal{G}=S^{\prime} \mathcal{G}^{\prime}$. The uniqueness of the decomposition of an $S L(2, \mathbb{Z})$ matrix into the product of a $\Gamma_{T}$ matrix and the representative of the coset $\Gamma_{T} \backslash S L(2, \mathbb{Z})$ then tells us that we must have $\mathcal{G}=\mathcal{G}^{\prime}$ and $S^{\prime}=I_{2}$. This establishes that the orbits associated with the different matrices of the form given in (4.5) are distinct. Therefore for a given choice of equivalences classes for the quadratic forms (2.5) and (2.6), represented by $q_{1}$ and $q_{2}$, the different duality orbits are labelled by minor matrices $M(\mathcal{A})$ of the form:

$$
M(\mathcal{A})=\mathcal{G}\left(\begin{array}{ll}
a & b \\
0 & d
\end{array}\right) M_{0}, \quad \mathcal{G} \in \Gamma_{T} \backslash S L(2, \mathbb{Z})
$$

for some reference minor matrix $M_{0}$ associated with the Bhargava cube for which the pair of quadratic forms are $q_{1} / m$ and $q_{2} / m$.

In special cases, when either $q_{1}$ is invariant under a subgroup of $\Gamma_{S}$ and/or $q_{2}$ is invariant under a subgroup of $\Gamma_{U}$, there may be further identification of the duality orbits as in $\$ 3$, This means that $(\mathcal{G}, a, b, d)$ and $\left(\mathcal{G}^{\prime}, a^{\prime}, b^{\prime}, d^{\prime}\right)$ may be equivalent for some special quadratic forms. These special quadratic forms are not necessarily those proportional to $\left(x^{2}+y^{2}\right)$ and 
$\left(x^{2}+x y+y^{2}\right)$, but are related to these via $S L(2, \mathbb{Z})$ transformation. For example $\left(x^{2}+y^{2}\right)$ is invariant under $\left(\begin{array}{cc}0 & -1 \\ 1 & 0\end{array}\right)$, but this matrix may not be an element of $\Gamma_{S}$ or $\Gamma_{U}$. However if we transform the quadratic form to another by an $S L(2, \mathbb{Z})$ transformation matrix $K$ using (2.8), the symmetry transformation that preserves the new quadratic form will be conjugated by $K$ and may belong to $\Gamma_{S}$ or $\Gamma_{U}$ for suitable choice of $K$. To see this let us represent $(x, y)$ as a row vector and let $q((x, y))$ be one of the forms $x^{2}+y^{2}$ or $x^{2}+x y+y^{2}$ with symmetry generated by the $S L(2, \mathbb{Z})$ matrix $A$. Then we have $q((x, y))=q((x, y) A)$. We now define $\tilde{q}((x, y))=q((x, y) K)$ where $K \in S L(2, \mathbb{Z})$. Then we have,

$$
\tilde{q}((x, y))=q((x, y) K)=q((x, y) K A)=\tilde{q}\left((x, y) K A K^{-1}\right) .
$$

For generating inequivalent special quadratic forms we simply use for $K$ all representative elements of the coset $\Gamma_{S} \backslash S L(2, \mathbb{Z})$ for $q_{1}$ and $\Gamma_{U} \backslash S L(2, \mathbb{Z})$ for $q_{2}$ and pick only those candidates for which $K A K^{-1}$ belongs to $\Gamma_{S}$ and $\Gamma_{U}$ respectively.

Once we have identified the special $q_{1}$ and $q_{2}$ candidates, we can repeat the analysis of $\oint_{3}$ to find out which of the duality orbits, labelled by $\mathcal{G}, a, b, d$, are the same. We shall now show that the results of $\$ 3$ can be used to solve this problem. First consider the case where only one of $q_{1}$ or $q_{2}$ is special. Let us for definiteness suppose that $q_{1}$ is special. In that case there are certain $\Gamma_{S}$ transformations which leave $q_{1}$ invariant. As in $\oint_{3}$ we can represent this by some $4 \times 4$ matrix $V$ multiplying $M_{0}$ from the right and this, in turn, can be transformed into an $S L(2, \mathbb{Z})$ matrix $\widetilde{S}\left(M_{0}, V\right)$ multiplying $M_{0}$ from the left. For the new Bhargava cube $\mathcal{A}^{\prime}$, related to the original Bhargava cube $\mathcal{A}$ by a duality transformation $V$ in $\Gamma_{S}$, (4.8) is replaced by

$$
M\left(\mathcal{A}^{\prime}\right)=\mathcal{G}\left(\begin{array}{ll}
a & b \\
0 & d
\end{array}\right) M_{0} V=\mathcal{G}\left(\begin{array}{ll}
a & b \\
0 & d
\end{array}\right) \widetilde{S}\left(M_{0}, V\right) M_{0}, \quad \mathcal{G} \in \Gamma_{T} \backslash S L(2, \mathbb{Z}) .
$$

Now, following the same arguments as in Appendix E, one can show that $\widetilde{S}\left(M_{0}, V\right)$ either squares to $-I_{2}$ or cubes to $-I_{2}$. and so, by appropriately redefining $M_{0}$, the matrix $\widetilde{S}\left(M_{0}, V\right)$ may be taken as one of the three forms given in (3.22) 8 We can now follow the same procedure described in (3.23)-(3.32) to solve for $\alpha, \beta, \gamma, \delta, a^{\prime}, b^{\prime}, d^{\prime}$ satisfying the equation:

$$
\left(\begin{array}{ll}
a & b \\
0 & d
\end{array}\right) \widetilde{S}\left(M_{0}, V\right)=\left(\begin{array}{ll}
\alpha & \beta \\
\gamma & \delta
\end{array}\right)\left(\begin{array}{ll}
a^{\prime} & b^{\prime} \\
0 & d^{\prime}
\end{array}\right)
$$

\footnotetext{
${ }^{8} \mathrm{As}$ in 93 , we are not including in this list the matrix $-I_{2}$, or the matrices related to (3.22) by a change in sign. However, if $-I_{2}$ is not an element of $\Gamma_{T}$, then we have to include these matrices in our list, since we can no longer absorb $-I_{2}$ into $S$ in (3.21). For example, if either $\Gamma_{S}$ or $\Gamma_{U}$ includes $-I_{2}$ but $\Gamma_{T}$ does not include it, then, since every quadratic form is invariant under $-I_{2}, \mathcal{G} \in \Gamma_{T} \backslash S L(2, \mathbb{Z})$ and $-\mathcal{G} \in \Gamma_{T} \backslash S L(2, \mathbb{Z})$ will give the same duality orbit even though they represent different elements of the coset.
} 
and then represent $\mathcal{G}\left(\begin{array}{ll}\alpha & \beta \\ \gamma & \delta\end{array}\right)$ as a $\Gamma_{T}$ element multiplied by a possibly new element $\mathcal{G}^{\prime}$ of $\Gamma_{T} \backslash S L(2, \mathbb{Z})$. This will tell us that the orbits labelled by $(\mathcal{G}, a, b, d)$ and $\left(\mathcal{G}^{\prime}, a^{\prime}, b^{\prime}, d^{\prime}\right)$ are the same. Note that even in the primitive case where $(a, b, d)=(1,0,1)$, this procedure may relate duality orbits labelled by different elements $\mathcal{G}$ and $\mathcal{G}^{\prime}$ of $\Gamma_{T} \backslash S L(2, \mathbb{Z})$. We shall see an example of this below.

Now consider the case where both $q_{1}$ and $q_{2}$ are special. Then both $q_{1}$ and $q_{2}$ correspond to some $S L(2, \mathbb{Z})$ transformation of $m \bar{q}(x, y)$ where $m$ is an integer and $\bar{q}(x, y)$ is either $\left(x^{2}+y^{2}\right)$ or $\left(x^{2}+x y+y^{2}\right)$, but the $S L(2, \mathbb{Z})$ transformations may not be the same. In any case, regarding these $S L(2, \mathbb{Z})$ transformations as $S L(2, \mathbb{Z})_{S}$ and $S L(2, \mathbb{Z})_{U}$ transformations, we can represent the combined transformation as a multiplication of the minor matrix $M(\mathcal{A})$ from the right by a $4 \times 4$ matrix $W \in S O(2,2 ; \mathbb{Z})$. Therefore if $\bar{M}_{0}$ denotes a choice of the minor matrix for the pair of quadratic forms $(\bar{q}(x, y), \bar{q}(x, y))$, then the minor matrix associated with the pair of quadratic forms $\left(q_{1} / m, q_{2} / m\right)$ can be taken to be

$$
M_{0}=\bar{M}_{0} W
$$

Furthermore the $\Gamma_{S} \times \Gamma_{U}$ transformation under which $\left(q_{1}, q_{2}\right)$ is invariant will be given by $V=W^{-1} \bar{V} W$ where $\bar{V}$ is an $S L(2, \mathbb{Z})_{S} \times S L(2, \mathbb{Z})_{U}$ transformation that leaves invariant the pair of quadratic forms $(\bar{q}(x, y), \bar{q}(x, y))$. Therefore, we have

$$
M_{0} V=\bar{M}_{0} W W^{-1} \bar{V} W=\widetilde{S}\left(\bar{M}_{0}, \bar{V}\right) \bar{M}_{0} W=\widetilde{S}\left(\bar{M}_{0}, \bar{V}\right) M_{0}
$$

where in the third step we have used $\bar{M}_{0} \bar{V}=\widetilde{S}\left(\bar{M}_{0}, \bar{V}\right) \bar{M}_{0}$. Therefore $\widetilde{S}\left(M_{0}, V\right)=\widetilde{S}\left(\bar{M}_{0}, \bar{V}\right)$. However we have seen in Appendix E that that $\widetilde{S}\left(\bar{M}_{0}, \bar{V}\right)$ is one of the three matrices appearing in (3.22). Therefore we can again use the procedure described in the previous paragraph to identify which sets of $(\mathcal{G}, a, b, d)$ correspond to the same duality orbit.

Since the special quadratic forms, for which we have additional identification of orbits as described above, are invariant under finite order elements of $\Gamma_{S}$ and/or $\Gamma_{U}$, we shall recall a few results on finite order elements of congruence subgroups of $S L(2, \mathbb{Z})$. It is known for example that $\Gamma(m)$ has no finite order elements for $m \geq 2, \Gamma_{1}(m)$ has no finite order elements for $m \geq 4$ and $\Gamma_{0}(m)$ has no finite order elements if -1 and -3 are not squares modulo $m$ [29]. Therefore if $\Gamma_{S}$ and $\Gamma_{U}$ are picked from these congruence subgroups of $S L(2, \mathbb{Z})$, then we have no special quadratic forms that are left invariant under any element of $\Gamma_{S}$ or $\Gamma_{U}$, and we do not have any additional identification of duality orbits of the kind described above. 
We shall now discuss some examples. If $\Gamma_{T}=\Gamma_{0}(2)$, then the representative elements of the coset $\Gamma_{T} \backslash S L(2, \mathbb{Z})$ may be taken as:

$$
\left(\begin{array}{ll}
1 & 0 \\
0 & 1
\end{array}\right), \quad\left(\begin{array}{cc}
0 & 1 \\
-1 & 0
\end{array}\right), \quad\left(\begin{array}{ll}
1 & 0 \\
1 & 1
\end{array}\right)
$$

In that case different possible choices of $g$ given in (4.5) may be taken to be:

$$
\left(\begin{array}{ll}
a & b \\
0 & d
\end{array}\right), \quad\left(\begin{array}{cc}
0 & d \\
-a & -b
\end{array}\right), \quad\left(\begin{array}{cc}
a & b \\
a & b+d
\end{array}\right), \quad a d=m, \quad 0 \leq b \leq d-1
$$

We see that as long as $a \neq 0$, which in turn follows from $a d=m \neq 0$, the three sets of matrices given in (4.15) are distinct. The minor matrix $M(\mathcal{A})$ associated with different orbits take the form:

$$
\left(\begin{array}{ll}
a & b \\
0 & d
\end{array}\right) M_{0}, \quad\left(\begin{array}{cc}
0 & d \\
-a & -b
\end{array}\right) M_{0}, \quad\left(\begin{array}{cc}
a & b \\
a & b+d
\end{array}\right) M_{0}, \quad a d=m, \quad 0 \leq b \leq d-1 .
$$

If on the other hand, either $\Gamma_{S}$ or $\Gamma_{U}$ is $\Gamma_{0}(2)$, then we have to classify the quadratic forms (2.5) and/or (2.6) by their $\Gamma_{0}(2)$ equivalence classes, and also identify the special quadratic forms for which there are additional identification of duality orbits. The special quadratic forms for $\Gamma_{0}(2)$ can be found as follows. As discussed above, they will be related to $x^{2}+y^{2}$ and $x^{2}+y^{2}+x y$ by appropriate elements of $S L(2, \mathbb{Z})$. To list the inequivalent ones, we can restrict our analysis to the elements of the coset (4.14). Consider first the quadratic form $x^{2}+y^{2}$ that is invariant under $\left(\begin{array}{cc}0 & -1 \\ 1 & 0\end{array}\right)$. This matrix is not in $\Gamma_{0}(2)$. It is easy to check that when we conjugate this by the second element of (4.14), the resulting matrix is still not in $\Gamma_{0}(2)$, but when we conjugate this by the third element of the coset (4.14) we get the matrix $\left(\begin{array}{ll}1 & -1 \\ 2 & -1\end{array}\right)$ which is in $\Gamma_{0}(2)$. The corresponding quadratic form, obtained by transforming $x^{2}+y^{2}$ by the third element of (4.14), is $x^{2}+2 x y+2 y^{2}$. One can also check that the conjugation of $\left(\begin{array}{cc}1 & -1 \\ 1 & 0\end{array}\right)$, which is the symmetry of $x^{2}+x y+y^{2}$, by any of the elements of the coset (4.14) does not produce a $\Gamma_{0}(2)$ matrix. Therefore we conclude that for $\Gamma_{0}(2), x^{2}+2 x y+2 y^{2}$ is the only special quadratic form up to $\Gamma_{0}(2)$ transformations. It follows from our argument above that when the quadratic forms (2.5) and/or (2.6) is proportional to $x^{2}+2 x y+2 y^{2}$, then in the classification of the orbits by $(\mathcal{G}, a, b, d)$ with $\mathcal{G} \in \Gamma_{T} \backslash S L(2, \mathbb{Z}), a, b, d \in \mathbb{Z}, 0 \leq b \leq d-1$, we need to identify $(\mathcal{G}, a, b, d)$ and $\left(\mathcal{G}^{\prime}, a^{\prime}, b^{\prime}, d^{\prime}\right)$, where the relation between $a, b, d$ and $a^{\prime}, b^{\prime}, d^{\prime}$ is given in (3.25) and the relation between $\mathcal{G}$ and $\mathcal{G}^{\prime}$ can be found by following the procedure described below (4.11). 
We shall now give an example to illustrate how for the special quadratic forms, the identification of the duality orbits labelled by $(\mathcal{G}, a, b, d)$ and $\left(\mathcal{G}^{\prime}, a^{\prime}, b^{\prime}, d^{\prime}\right)$ needs to be taken into account even for primitive quadratic forms for which $(a, b, d)=\left(a^{\prime}, b^{\prime}, d^{\prime}\right)=(1,0,1)$. For this let us suppose that the duality group is $S L(2, \mathbb{Z})_{S} \times \Gamma_{0}(2)_{T} \times S L(2, \mathbb{Z})_{U}$, and that the quadratic form $q_{1}$ given in (2.5), transforming under $S L(2, \mathbb{Z})_{S}$, is proportional to $x^{2}+y^{2}$. The choice of $q_{2}$ is arbitrary, but we take this to be coprime to $q_{1}$. Then we have $a=1, b=0, d=1$ and for given $q_{1}, q_{2}$, the duality orbits are labelled by the representative elements of the coset $\Gamma_{T} \backslash S L(2, \mathbb{Z})=\Gamma_{0}(2) \backslash S L(2, \mathbb{Z})$. These have been listed in (4.14). We now apply (4.11) to determine which duality orbits should be identified. Since $\left(\begin{array}{ll}a & b \\ 0 & d\end{array}\right)$ is the identity matrix, we can get a solution to (4.11) by setting

$$
\left(\begin{array}{ll}
\alpha & \beta \\
\gamma & \delta
\end{array}\right)=\widetilde{S}\left(M_{0}, V\right)=\left(\begin{array}{cc}
0 & -1 \\
1 & 0
\end{array}\right), \quad\left(\begin{array}{ll}
a^{\prime} & b^{\prime} \\
0 & d^{\prime}
\end{array}\right)=\left(\begin{array}{ll}
1 & 0 \\
0 & 1
\end{array}\right) .
$$

Therefore the new coset element $\mathcal{G}^{\prime}$ is given by

$$
\mathcal{G}^{\prime}=\mathcal{G}\left(\begin{array}{ll}
\alpha & \beta \\
\gamma & \delta
\end{array}\right)=\mathcal{G}\left(\begin{array}{cc}
0 & -1 \\
1 & 0
\end{array}\right)
$$

up to multiplication by $\Gamma_{0}(2)$ matrices from the left. It is now easy to see that this exchanges the first two coset representatives in (4.14), leaving the third one unchanged. Therefore for a pair of coprime quadratic forms, when one of the quadratic forms is proportional to $x^{2}+y^{2}$, we need to identify the duality orbits labelled by first two elements of (4.14).

The reader would have noticed that in our analysis we treat $\Gamma_{S}$ and $\Gamma_{U}$ differently from $\Gamma_{T}$. However since we are classifying the duality orbits of $\Gamma_{S} \times \Gamma_{T} \times \Gamma_{U}$, the final result should be symmetric in all three groups. We verify this in Appendix $\mathrm{F}$ by counting the number of duality orbits in several examples.

So far we have assumed that the charge lattice is the lattice of integers. However in specific string theory models, only a sublattice of the lattice of integers represents allowed charges. In such cases many of the duality orbits described above will remain empty. For example for the STU model provided by example D of [13], the charge lattice has the following form:

$$
Q_{1} \in \mathbb{Z} / 2, \quad Q_{2}, Q_{3}, Q_{4} \in \mathbb{Z}, \quad P_{3} \in 2 \mathbb{Z}, \quad P_{1}, P_{2}, P_{4} \in \mathbb{Z}
$$

It can be checked that this lattice is invariant under $\Gamma_{0}(2)_{S} \times \Gamma_{0}(2)_{T} \times \Gamma_{0}(2)_{U}$ duality group with the $\Gamma_{0}(2)$ actions as given in (2.1)-(2.3), with $\alpha, \delta$ odd, $\gamma$ even and $\beta$ arbitrary integer. However in this form the charge lattice is not integral. We can rectify this by redefining the $Q_{i}$ 's 
by mutiplying them by a factor of 2 . This does not change the $\mathrm{U}$ and T-duality transformation rules given in (2.1) and (2.2), but the S-duality transformation given in (2.3) now becomes a $\Gamma^{0}(2)$ transformation. We can make this into a $\Gamma_{0}(2)$ transformation by relabelling $Q_{i}$ 's as $P_{i}$ 's and $P_{i}$ 's as $Q_{i}$ 's. Therefore the restriction on the charges given in (4.19) now translates to:

$$
P_{1} \in \mathbb{Z}, \quad P_{2}, P_{3}, P_{4} \in 2 \mathbb{Z}, \quad Q_{3} \in 2 \mathbb{Z}, \quad Q_{1}, Q_{2}, Q_{4} \in \mathbb{Z} .
$$

If we compute the quadratic forms given in (2.5), (2.6) and (2.7) with these charges, then the coefficients of $y^{2}$ and $x y$ are always even. In our classification scheme, this already eliminates all duality orbits where one of these coefficients is odd, either for (2.5) or for (2.6). Furthermore, for a given choice of the reference matrix $M_{0}$, the choice of $a, b, d$ in (4.16) will be restricted so that $M(\mathcal{A})$ given in (4.16) is compatible with the charge quantization condition after using (3.2).

We shall illustrate this with an example. It can be easily checked that given the restriction (4.20) on the charges, even and odd $P_{1}$ charges do not mix with each other under $\Gamma_{0}(2)_{S}, \Gamma_{0}(2)_{T}$ and $\Gamma_{0}(2)_{U}$ transformations given in (2.1) $-(2.3)$. Let us consider the case where $P_{1} \in 2 \mathbb{Z}+1$. In this case, it is easy to see that only one of the three matrices in (4.16) will satisfy the condition $P_{1} \in 2 \mathbb{Z}+1, P_{4} \in 2 \mathbb{Z}$. For example let us suppose that the first matrix generates a charge vector for which $P_{1}=2 k+1, P_{4}=2 \ell$ with $k, \ell \in \mathbb{Z}$. Then the second matrix in (4.16) will generate $P_{1}=2 \ell, P_{4}=-2 k-1$ and the third matrix in (4.16) will generate $P_{1}=(2 k+1)$, $P_{4}=2 k+2 \ell+1$. Both of these violate the condition $P_{1} \in 2 \mathbb{Z}+1, P_{4} \in 2 \mathbb{Z}$. Therefore for the subset of charges for which $P_{1} \in 2 \mathbb{Z}+1$, the $\Gamma_{0}(2)$ equivalence classes of $q_{1}, q_{2}$ and the integers $a, b, d$ fix the duality orbit. Of course when one of the quadratic forms is special, we have further identification of duality orbits. For $P_{1} \in 2 \mathbb{Z}$ we do not have such simplifications and we need to include the orbits given by different matrices in (4.16).

There are also other versions of the STU model with different charge lattice and the duality group given by other congruence subgroups of $S L(2, \mathbb{Z})$.

In some cases, when two or all of the groups $\Gamma_{S}, \Gamma_{T}$ and $\Gamma_{U}$ are identical congruence subgroups of $S L(2, \mathbb{Z})$, the relevant duality group may also include some extra elements that do not commute with $\Gamma_{S}, \Gamma_{T}$ and $\Gamma_{U}$, but permute them under conjugation. In such cases there will be additional identification among the duality orbits classified above, that are related by the action of these extra elements. We shall not discuss this, but the details can be easily worked out. An example of this is provided in heterotic string theory compactified on $T^{6}$ if we consider an appropriately twisted index where the twist includes a change of sign of four of 
the circles of $T^{6}$ and of the lattice associated with the gauge group $E_{8} \times E_{8}$ or $\operatorname{Spin}(32) / \mathbb{Z}_{2}$. In this case the only charges that are invariant under this twist are the momentum and winding numbers along the two remaining circles of $T^{6}$ and their magnetic counterparts. The duality group that acts non-trivially on these charges is $S L(2, \mathbb{Z})_{S} \times O(2,2 ; \mathbb{Z})$ which can also be regarded as $S L(2, \mathbb{Z})_{S} \times S L(2 ; \mathbb{Z})_{T} \times S L(2, \mathbb{Z})_{U} \rtimes \mathbb{Z}_{2}$, where the $\mathbb{Z}_{2}$ exchanges $Q_{1}$ and $Q_{3}$. It can be seen from (2.1) and (2.2) that this exchanges the $S L(2, \mathbb{Z})_{U}$ and $S L(2, \mathbb{Z})_{T}$ action on the charges. Hence in this case, the duality orbit (for primitive quadratic forms) is specified by two equivalence classes, associated with the left-right and the front-back faces of the Bhargava cube shown in Fig. 1, with an additional equivalence relation that exchanges the two equivalence classes.

Acknowledgement: We wish to thank Shamit Kachru for comments on the manuscript and for raising some interesting questions. NB, SD and RKS would like to acknowledge the hospitality of HRI where part of the work was done. AS would like to acknowledge the hospitality of IISER Bhopal where this work began. The work of NB is supported by SERB ECR grant 2019-2022. NB would like to thank Regular Associateship, the Abdus Salam ICTP, Trieste, Italy. The work of AB is supported by the grant no. MTR/2019/000582 from the SERB, Government of India. The work of SD is supported by the grant no. EMR/2016/006294 and MTR/2019/000390 from the SERB, Government of India. SD also acknowledges the Simons Associateship of the Abdus Salam ICTP, Trieste, Italy. The work of AS was supported in part by the J. C. Bose fellowship of the Department of Science and Technology, India and also by the Infosys Chair Professorship. Finally, we are grateful to people of India for their unconditional support towards researches in basic sciences.

\section{A Proof of Gauss's Lemma}

In this appendix we shall describe the proof of Gauss's Lemma following [10]:

Lemma A.1. Let

$$
M=\left(\begin{array}{llll}
p_{1} & p_{2} & \ldots & p_{n} \\
r_{1} & r_{2} & \ldots & r_{n}
\end{array}\right) \quad \text { and } \quad M^{\prime}=\left(\begin{array}{cccc}
p_{1}^{\prime} & p_{2}^{\prime} & \ldots & p_{n}^{\prime} \\
r_{1}^{\prime} & r_{2}^{\prime} & \ldots & r_{n}^{\prime}
\end{array}\right)
$$

be two $2 \times n$ integer matrices ( $n \geq 3$ ) with the following properties:

1. the $2 \times 2-$ minors of $M$ are coprime; 
2. there is an integer $m$ such that each minor of $M^{\prime}$ is $m$ times the corresponding minor of $M$.

Then there is a matrix $g=\left(\begin{array}{ll}a & b \\ c & d\end{array}\right)$ with determinant $m$ and $a, b, c, d \in \mathbb{Z}$ such that $M^{\prime}=A M$.

Proof. Since the minors $M_{i k}=\left|\begin{array}{ll}p_{i} & p_{k} \\ r_{i} & r_{k}\end{array}\right|=-M_{k i}$ are coprime, there exists $n(n-1) / 2$ integers $x_{i k}$ for $i<k$ such that

$$
\sum_{\substack{i, k=1 \\
i<k}}^{n} x_{i k}\left|\begin{array}{cc}
p_{i} & p_{k} \\
r_{i} & r_{k}
\end{array}\right|=1
$$

Now we have

$$
\begin{aligned}
p_{k}\left|\begin{array}{cc}
p_{i}^{\prime} & p_{j}^{\prime} \\
r_{i} & r_{j}
\end{array}\right|+r_{k}\left|\begin{array}{cc}
p_{i} & p_{j} \\
p_{i}^{\prime} & p_{j}^{\prime}
\end{array}\right| & =p_{k} p_{i}^{\prime} r_{j}-p_{k} p_{j}^{\prime} r_{i}+r_{k} p_{i} p_{j}^{\prime}-r_{k} p_{j} p_{i}^{\prime} \\
& =p_{i}^{\prime}\left(p_{k} r_{j}-r_{k} p_{j}\right)-p_{j}^{\prime}\left(p_{k} r_{i}-p_{i} r_{k}\right) \\
& =\frac{1}{m}\left\{p_{i}^{\prime}\left(p_{k}^{\prime} r_{j}^{\prime}-r_{k}^{\prime} p_{j}^{\prime}\right)-p_{j}^{\prime}\left(p_{k}^{\prime} r_{i}^{\prime}-p_{i}^{\prime} r_{k}^{\prime}\right)\right\} \\
& =\frac{1}{m} p_{k}^{\prime}\left|\begin{array}{cc}
p_{i}^{\prime} & p_{j}^{\prime} \\
r_{i}^{\prime} & r_{j}^{\prime}
\end{array}\right|=p_{k}^{\prime}\left|\begin{array}{cc}
p_{i} & p_{j} \\
r_{i} & r_{j}
\end{array}\right|
\end{aligned}
$$

Similarly, we have,

$$
p_{k}\left|\begin{array}{cc}
r_{i}^{\prime} & r_{j}^{\prime} \\
r_{i} & r_{j}
\end{array}\right|+r_{k}\left|\begin{array}{cc}
p_{i} & p_{j} \\
r_{i}^{\prime} & r_{j}^{\prime}
\end{array}\right|=r_{k}^{\prime}\left|\begin{array}{cc}
p_{i} & p_{j} \\
r_{i} & r_{j}
\end{array}\right|
$$

Let us define

$$
\begin{array}{ll}
a=\sum_{\substack{i, j \\
i<j}} x_{i j}\left|\begin{array}{cc}
p_{i}^{\prime} & p_{j}^{\prime} \\
r_{i} & r_{j}
\end{array}\right|, \quad b=\sum_{\substack{i, j \\
i<j}} x_{i j}\left|\begin{array}{cc}
p_{i} & p_{j} \\
p_{i}^{\prime} & p_{j}^{\prime}
\end{array}\right|, \\
c=\sum_{\substack{i, j \\
i<j}} x_{i j}\left|\begin{array}{cc}
r_{i}^{\prime} & r_{j}^{\prime} \\
r_{i} & r_{j}
\end{array}\right|, & d=\sum_{\substack{i, j \\
i<j}} x_{i j}\left|\begin{array}{cc}
p_{i} & p_{j} \\
r_{i}^{\prime} & r_{j}^{\prime}
\end{array}\right| .
\end{array}
$$

Then using (A.2), (A.3), (A.5) wet,

$$
a p_{k}+b r_{k}=\sum_{\substack{i, j \\
i<j}} x_{i j} p_{k}^{\prime}\left|\begin{array}{cc}
p_{i} & p_{j} \\
r_{i} & r_{j}
\end{array}\right|=p_{k}^{\prime} \sum_{\substack{i, j \\
i<j}} x_{i j}\left|\begin{array}{cc}
p_{i} & p_{j} \\
r_{i} & r_{j}
\end{array}\right|=p_{k}^{\prime}
$$

Similarly we have $c p_{k}+d r_{k}=r_{k}^{\prime}$. Thus we have that

$$
\left(\begin{array}{l}
p_{k}^{\prime} \\
r_{k}^{\prime}
\end{array}\right)=g\left(\begin{array}{l}
p_{k} \\
r_{k}
\end{array}\right), \quad g=\left(\begin{array}{ll}
a & b \\
c & d
\end{array}\right)
$$

This gives $M^{\prime}=g M$. Finally from the relation $M_{i j}^{\prime}=\operatorname{det}(g) M_{i j}$, obtained from the relation $M^{\prime}=g M$, and hypothesis 2 , we conclude that $\operatorname{det}(g)=m$. 


\section{B Proof of existence of a Bhargava cube for a given pair of quadratic forms}

In this appendix we shall prove, following [10,11], the existence of a Bhargava cube for any given pair of quadratic forms of the same discriminant, even when they are not primitive.

Theorem B.1. Let $q_{i}(x, y)=A_{i} x^{2}+B_{i} x y+C_{i} y^{2}, \quad i=1,2$ be two quadratic forms of same discriminant $D$. Then there exists a Bhargava cube with $q_{T}=q_{1}$ and $q_{L}=q_{2}$, where $q_{T}$ is the quadratic form associated with the top-down faces of the cube and $q_{L}$ is the quadratic form associated with the left-right faces of the cube.

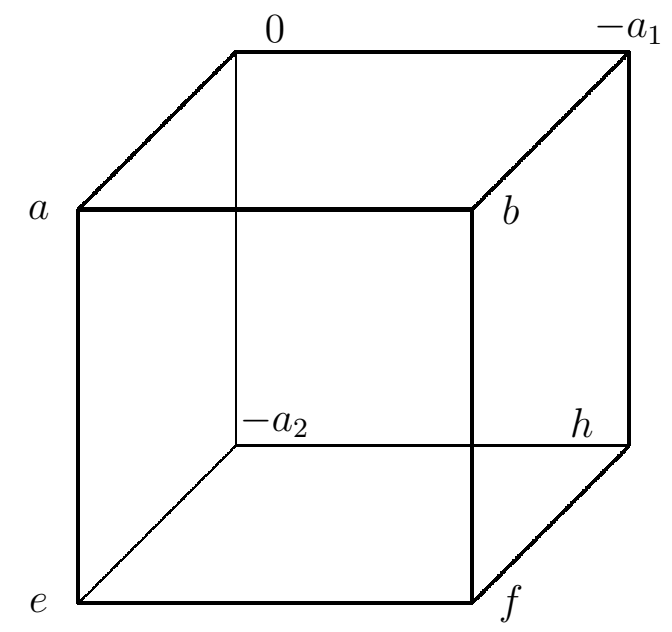

Figure 2: Bhargava cube for $q_{1}$ and $q_{2}$

Proof. We will construct a Bhargava cube of the form shown in Figure 2, The quadratic forms associated with the top-down and left-right faces of the cube are given by:

$$
\begin{aligned}
& q_{T}(x, y)=\left(a a_{1}\right) x^{2}-\left(a h-a_{1} e+a_{2} b\right) x y-\left(e h+a_{2} f\right) y^{2}, \\
& q_{L}(x, y)=\left(a a_{2}\right) x^{2}-\left(a h+a_{1} e-a_{2} b\right) x y-\left(b h+a_{1} f\right) y^{2} .
\end{aligned}
$$

Equating the $x^{2}$ coefficients in $\left(q_{1}, q_{2}\right)$ with those in $\left(q_{T}, q_{L}\right)$, and the $x y$ coefficient in $q_{1}+q_{2}$ with that in $q_{T}+q_{L}$, we get,

$$
A_{1}=a a_{1}, \quad A_{2}=a a_{2}, \quad \frac{B_{1}+B_{2}}{2}=-a h .
$$

Therefore we can solve (B.2) by taking

$$
a=\operatorname{gcd}\left(A_{1}, A_{2},\left(B_{1}+B_{2}\right) / 2\right), \quad a_{1}=A_{1} / a, \quad a_{2}=A_{2} / a, \quad h=-\left(B_{1}+B_{2}\right) / 2 a .
$$


This gives

$$
\operatorname{gcd}\left(a_{1}, a_{2}, h\right)=1 .
$$

We note, however, that for $a>1$, other choices are also possible. Equating the $y^{2}$ coefficients in $\left(q_{1}, q_{2}\right)$ with those in $\left(q_{T}, q_{L}\right)$, we get

$$
h e+a_{2} f=-C_{1}, \quad h b+a_{1} f=-C_{2} .
$$

Finally, equating the coefficient of $x y$ in $q_{1}-q_{2}$ with that in $q_{T}-q_{L}$ we get,

$$
a_{1} e-a_{2} b=\frac{B_{1}-B_{2}}{2} .
$$

Note that once (B.6) is satisfied, only one of the two equations in $(\overline{B .5})$ is independent. This is due to the fact that the since the discriminant of $q_{1}$ and $q_{2}$ are equal, once $A_{1}, A_{2}, B_{1}$ and $B_{2}$ have been fixed, $C_{1}$ is given in terms of $C_{2}$ and vice versa.

Equality of the discriminants of $q_{1}$ and $q_{2}$ give

$$
B_{1}^{2}-4 A_{1} C_{1}=B_{2}^{2}-4 A_{2} C_{2} \Longrightarrow \frac{B_{1}-B_{2}}{2}=\frac{A_{1} C_{1}-A_{2} C_{2}}{\left(B_{1}+B_{2}\right) / 2}=-\frac{\left(a_{1} C_{1}-a_{2} C_{2}\right)}{h} .
$$

Using (B.4) and (B.7), we get

$$
\operatorname{gcd}\left(a_{1}, a_{2}\right) \mid \frac{\left(B_{1}-B_{2}\right)}{2} .
$$

This in turn shows that (B.6) admits integer solutions for $e$ and $b$. If $\left(e_{0}, b_{0}\right)$ denotes a particular pair of solutions, then the general solution takes the form:

$$
b=b_{0}+r a_{1} / \operatorname{gcd}\left(a_{1}, a_{2}\right), \quad e=e_{0}+r a_{2} / \operatorname{gcd}\left(a_{1}, a_{2}\right), \quad r \in \mathbb{Z} .
$$

We now substitute this into (B.5) to find $f$. As discussed above, it is sufficient to solve one of these equations, since the other will then follow by the equality of the discriminants of $q_{1}$ and $q_{2}$. Therefore we can express the solution as,

$$
f=-\frac{1}{a_{2}}\left\{C_{1}+h e_{0}+h a_{2} r / \operatorname{gcd}\left(a_{1}, a_{2}\right)\right\}, \quad \text { or } \quad f=-\frac{1}{a_{1}}\left\{C_{2}+h b_{0}+h a_{1} r / \operatorname{gcd}\left(a_{1}, a_{2}\right)\right\} .
$$

The equality of the two solutions follows from the fact that $\left(e_{0}, b_{0}\right)$ solves (B.6). Let us define $q=\operatorname{gcd}\left(a_{1}, a_{2}\right)$. Now for general choice of $r \in \mathbb{Z}, f$ is not an integer. But from (B.10) we can see that $a_{2}(f+h r / q)$ and $a_{1}(f+h r / q)$ are integers. Therefore, the denominator of $(f+h r / q)$ must divide $a_{1}$ and $a_{2}$ and hence must divide $q=\operatorname{gcd}\left(a_{1}, a_{2}\right)$. This allows us to 
write $(f+h r / q)=p / q$ after appropriately multiplying the numerator and denominator of $f$ by an integer if necessary. This gives:

$$
f=\frac{p-h r}{\operatorname{gcd}\left(a_{1}, a_{2}\right)}, \quad p \in \mathbb{Z} .
$$

We now need to find an $r$ that makes $f$ an integer. This is possible if $\operatorname{gcd}\left(h, \operatorname{gcd}\left(a_{1}, a_{2}\right)\right)=1$. The latter equation follows from (B.4).

This proves the existence of a Bhargava cube satisfying the constraints $q_{T}=q_{1}$ and $q_{L}=q_{2}$ for any pair of binary quadratic forms $q_{1}$ and $q_{2}$.

Note that we nowhere assumed that the quadratic forms $q_{1}$ and/or $q_{2}$ are primitive. We have also not assumed that $q_{1}, q_{2}$ are coprime. We shall end this appendix by giving some examples of this construction for non-primitive $q_{1}, q_{2}$. Instead of giving the Bhargava cube, we shall give the minor matrix associated with the cube shown in Fig. 2.

$$
\left(\begin{array}{cccc}
a & b & e & f \\
0 & -a_{1} & -a_{2} & h
\end{array}\right)
$$

Consider the pair of quadratic forms:

$$
q_{1}=10\left(x^{2}+2 x y+10 y^{2}\right), \quad q_{2}=3 x^{2}+300 y^{2} .
$$

Here neither $q_{1}$ nor $q_{2}$ is primitive, although $q_{1}$ and $q_{2}$ are coprime. The corresponding minor matrix, constructed following the procedure described in this appendix, is:

$$
M(\mathcal{A})=\left(\begin{array}{cccc}
1 & 40 & 13 & 10 \\
0 & -10 & -3 & -10
\end{array}\right)
$$

Note that $M(\mathcal{A})$ is not unique, but requires a choice of $r$ in $(\overline{\mathrm{B} .9}),(\overline{\mathrm{B} .11})$, which is determined up to shift by integer multiples of $\operatorname{gcd}\left(a_{1}, a_{2}\right)$. If instead we consider the pair of quadratic forms:

$$
q_{1}=10 m\left(x^{2}+2 x y+10 y^{2}\right), \quad q_{2}=m\left(3 x^{2}+300 y^{2}\right),
$$

which are not even coprime, we can still construct a minor matrix by the procedure described in this appendix:

$$
\left(\begin{array}{cccc}
m & 40 m & 13 m & 10 m \\
0 & -10 & -3 & -10
\end{array}\right)=\left(\begin{array}{cc}
m & 0 \\
0 & 1
\end{array}\right)\left(\begin{array}{cccc}
1 & 40 & 13 & 10 \\
0 & -10 & -3 & -10
\end{array}\right) .
$$

This is consistent with Gauss's Lemma. 
As another example, consider the pair of quadratic forms:

$$
q_{1}=4 x^{2}+4 x y+34 y^{2}, \quad q_{2}=12 x^{2}+12 x y+14 y^{2} .
$$

Note that neither $q_{1}$ nor $q_{2}$ are primitive and they are also not coprime. The corresponding minor matrix, constructed using the procedure described above, can be chosen to be:

$$
M(\mathcal{A})=\left(\begin{array}{cccc}
4 & 7 & 17 & 0 \\
0 & -1 & -3 & -2
\end{array}\right)=\left(\begin{array}{cc}
2 & 1 \\
0 & 1
\end{array}\right)\left(\begin{array}{cccc}
2 & 4 & 10 & 1 \\
0 & -1 & -3 & -2
\end{array}\right) .
$$

The minor matrix $\left(\begin{array}{cccc}2 & 4 & 10 & 1 \\ 0 & -1 & -3 & -2\end{array}\right)$ is the minor matrix associated with the quadratic forms $q_{1} / 2$ and $q_{2} / 2$. This is again consistent with Gauss's Lemma.

Note that since the minor matrix (B.12) always has a zero in its lower left corner, the matrix $g$ appearing in (3.10) will always have the form $\left(\begin{array}{ll}a & b \\ 0 & d\end{array}\right)$ for some integers $a, b$ and $d$. Furthermore, since we have chosen $a_{1}, a_{2}$ and $h$ to be coprime, $d$ must be 1 . This of course is a particular choice. For a given pair of quadratic form, we can generate matrices with $d \neq 1$ by modifying our algorithm and allowing $a_{1}, a_{2}$ and $h$ to have a common factor.

\section{Bhargava's results}

In this appendix we shall briefly review the relevant parts of Bhargava's results that we are using. Let $q_{1}$ and $q_{2}$ be a pair of primitive binary quadratic forms with the same discriminant. Then it follows, as a special case of the results of appendix $\mathrm{B}$, that there exists a Bhargava cube for which $q_{1}$ is the quadratic form associated with the top-down faces of the cube, and $q_{2}$ is the quadratic form associated with the left-right faces of the cube. Furthermore it follows from Gauss's Lemma with $m=1$ that if we consider two cubes with this property, then they are related by an $S L(2, \mathbb{Z})$ transformation acting on the entries connected by a front-back line. Therefore the quadratic form $q_{3}$ associated with the front-back face is determined uniquely up to an $S L(2, \mathbb{Z})$ transformation. This is the first part of the Bhargava's theorem that we have used in our analysis in $\$ 2$. Of course, Bhargava's result says more - that the product of the class group elements associated with the quadratic forms $q_{1}, q_{2}$ and $q_{3}$ is the identity element of the class group, but we have not used this result and will not discuss its proof.

The second part of Bhargava's results that we have used says that two different charge vectors, for which the corresponding Bhargava cubes have the same (primitive) class group 
elements, can be related to each other by a duality transformation. Let $\mathcal{A}$ and $\mathcal{A}^{\prime}$ be the Bhargava cubes associated with this pair of charge vectors, and let $\left(q_{1}, q_{2}, q_{3}\right)$ and $\left(q_{1}^{\prime}, q_{2}^{\prime}, q_{3}^{\prime}\right)$ be the corresponding quadratic forms. Since $q_{1}$ and $q_{1}^{\prime}$ describe the same class group elements, there is an $S L(2, \mathbb{Z})$ transformation that relates $q_{1}$ to $q_{1}^{\prime}$. Similar argument shows that there is an $S L(2, \mathbb{Z})$ transformation that relates $q_{2}$ to $q_{2}^{\prime}$. We apply these transformations on the entries of $\mathcal{A}$ connected by top-down and left-right lines respectively, producing a new Bhargava cube $\mathcal{A}^{\prime \prime}$. The cubes $\mathcal{A}^{\prime}$ and $\mathcal{A}^{\prime \prime}$ now have the same quadratic forms associated with the top-down and left-right faces. Therefore, by Gauss's Lemma, there is an $S L(2, \mathbb{Z})$ transformation, acting on the entries connected by front-back lines, that relate $\mathcal{A}^{\prime \prime}$ to $\mathcal{A}^{\prime}$. Combining the two duality transformations, one relating $\mathcal{A}$ to $\mathcal{A}^{\prime \prime}$ and the other relating $\mathcal{A}^{\prime \prime}$ to $\mathcal{A}^{\prime}$, we get the duality transformation that relates $\mathcal{A}$ to $\mathcal{A}^{\prime}$.

\section{Automorphisms of binary quadratic forms}

In this appendix we shall review some basic facts about automorphisms of binary quadratic forms (see [21,22] for details). We say that $K \in S L(2, \mathbb{Z})$ is a proper automorphism of a binary quadratic form $f=A x^{2}+B x y+C y^{2}$ if

$$
f(x, y)=f((x, y) K)
$$

The group of proper automorphisms of $f$ is denoted by $\operatorname{Aut}(f)$. We say that $f$ has a trivial proper automorphism group if $\operatorname{Aut}(f)=\left\{ \pm I_{2}\right\}$, where $I_{2}$ is the $2 \times 2$ identity matrix. Let $f=A x^{2}+B x y+C y^{2}$ be a primitive binary quadratic form with discriminant $D$. Consider the associated Pell equation [21,22]

$$
p^{2}-D q^{2}=4
$$

Given $(p, q) \in \mathbb{Z}^{2}$, we define

$$
K(f, p, q)=\left(\begin{array}{cc}
(p-q B) / 2 & q A \\
-q C & (p+q B) / 2
\end{array}\right)
$$

It is straightforward to verify that $K$ given in (D.3) satisfies (D.1). We also see that for any $D$, $p= \pm 2, q=0$ is a solution to $(\overline{\mathrm{D} .2})$, but this generates the trivial automorphisms $\pm I_{2}$. The following theorem gives a correspondence between solutions of Pell's equation and Aut $(f)$.

Theorem D.1. The map $(p, q) \mapsto K(f, p, q)$ is a bijection between the set of integer solutions of $p^{2}-D q^{2}=4$ and $\operatorname{Aut}(f)$. 
The following results will also be useful.

Theorem D.2. If $f$ and $g$ are primitive binary quadratic forms with the same discriminant, then $A u t(f) \cong A u t(g)$.

Theorem D.3. If $f$ is a primitive positive definite quadratic form with discriminant $D \neq$ $-3,-4$ then $A u t(f)$ is trivial.

This theorem tells us that the only cases we need to consider are $D=-3$ and $D=-4$. Let us introduce the matrices:

$$
W_{1}=\left(\begin{array}{cc}
0 & -1 \\
1 & 0
\end{array}\right), \quad W_{2}=\left(\begin{array}{cc}
1 & -1 \\
1 & 0
\end{array}\right) .
$$

(i) If $D=-4$ then by Theorem $\underline{\mathrm{D} .2}$, it suffices to consider the quadratic form $f_{-4}=x^{2}+y^{2}$. Then we have $(p, q)=( \pm 2,0)$ or $(0, \pm 1)$, and,

$$
\operatorname{Aut}\left(f_{-4}\right)=\left\{ \pm I_{2}, \pm W_{1}\right\}=\left\langle W_{1}\right\rangle
$$

which is a cyclic group of order 4 generated by $W_{1}$. Note that the other inequivalent quadratic form $-f_{-4}$ has the same automorphism group as $f_{-4}$.

(ii) Likewise, for $D=-3$ it suffices to consider the quadratic form $f_{-3}=x^{2}+x y+y^{2}$. In this case we have $(p, q)=( \pm 2,0)$ or $( \pm 1, \pm 1)$, and,

$$
\operatorname{Aut}\left(f_{-3}\right)=\left\langle W_{2}\right\rangle
$$

which is a cyclic group of order 6 generated by $W_{2}$.

For non-primitive quadratic form $f$ with a common factor $r \in \mathbb{Z}$, the restriction on the automrphism groups can be found by considering the quadratic form $f / r$ with discriminant $D / r^{2}$. The previous results tell us that $\operatorname{Aut}(f)$ is trivial unless $D=-3 r^{2}$ or $-4 r^{2}$, and is isomorphic to (D.5) for $D=-4 r^{2}$ and (D.6) for $D=-3 r^{2}$.

As described in (4.2), there is also a correspondence between positive definite binary quadratic forms and points in the upper half plane. Given a quadratic form $f=A x^{2}+B x y+C y^{2}$ of discriminant $D$, the correspondence is

$$
f \mapsto-\frac{B}{2 C}+i \frac{\sqrt{|D|}}{2|C|} .
$$

Using this, the form $x^{2}+y^{2}$ corresponds to the point $i$ and $x^{2}+x y+y^{2}$ corresponds to the point $\frac{-1+i \sqrt{3}}{2}=e^{2 \pi i / 3}$. 
One can use these results to restrict the possible automorphism groups of quadratic forms inside congruence subgroups. For example, for $D=-3$, we must have $B$ odd. Setting $B=2 k+1$ with $k \in \mathbb{Z}$, the equation $B^{2}-4 A C=-3$ reduces to $A C=k(k+1)+1$. Therefore $A$ and $C$ must be odd. Eq.(D.3) now shows that since for non-trivial automorphism $p= \pm 1$ and $q= \pm 1$, the off-diagonal elements of the matrix are both odd. This immediately rules out non-trivial Aut $(f)$ inside $\Gamma_{0}(N), \Gamma^{0}(N), \Gamma_{1}(N), \Gamma^{1}(N)$ and $\Gamma(N)$ for even $N$ and $D=-3$.

\section{E Possible forms of $\widetilde{S}\left(M_{0}, V\right)$}

In this appendix we shall find the possible forms of $\widetilde{S}\left(M_{0}, V\right)$ introduced in (3.20). Recall that $V$ represents an $S L(2, \mathbb{Z})_{S} \times S L(2, \mathbb{Z})_{U}$ transformation, represented by a $4 \times 4$ matrix, that leaves some quadratic form invariant. It follows from (D.5), (D.6) that in the range (2.10), the only examples of quadratic forms that are invariant under some $S L(2, \mathbb{Z})$ transformation of the form given in (2.8) are as follows [21]:9

$$
\begin{aligned}
& c\left(x^{2}+y^{2}\right) \text { is invariant under } \operatorname{Aut}\left(f_{-4}\right) \text { generated by } W_{1}, \\
& c\left(x^{2}+x y+y^{2}\right) \text { is invariant under } \operatorname{Aut}\left(f_{-3}\right) \text { generated by } W_{2},
\end{aligned}
$$

for any integer $c$. For a quadratic form proportional to $\left(x^{2}+y^{2}\right)$, the discriminant is $-4 p^{2}$ for $p \in \mathbb{Z}$ whereas for a quadratic form proportional to $\left(x^{2}+x y+y^{2}\right)$ the discriminant is $-3 q^{2}$ for $q \in \mathbb{Z}$. Since we cannot have $4 p^{2}=3 q^{2}$ for $p, q \in \mathbb{Z}$, we see that we cannot have $q_{1} \propto\left(x^{2}+y^{2}\right)$ and $q_{2} \propto\left(x^{2}+x y+y^{2}\right)$ or vice versa. Therefore we have to consider the cases where either only one of them is proportional to $\left(x^{2}+y^{2}\right)$ or $\left(x^{2}+x y+y^{2}\right)$ or both of them are proportional to $\left(x^{2}+y^{2}\right)$ or both of them are proportional to $\left(x^{2}+x y+y^{2}\right)$.

First consider the case where one of them is proportional to $\left(x^{2}+y^{2}\right)$. In this case, $W_{1}$ given in (D.4) (and its powers) is the symmetry of the quadratic form. This is represented by a $4 \times 4$ matrix $V$ multiplying $M_{0}$ from the right. The precise form of $V$ depends on whether $W_{1}$ is in $S L(2, \mathbb{Z})_{S}$ or $S L(2, \mathbb{Z})_{U}$, i.e. whether $q_{1}$ or $q_{2}$ is proportional to $\left(x^{2}+y^{2}\right)$, but since $W_{1}^{2}=-I_{2}$, we must have $V^{2}=-I_{4}$ where $I_{4}$ is the $4 \times 4$ identity matrix.10 It then follows from (3.20) that $\widetilde{S}\left(M_{0}, V\right)^{2}=-I_{2}$. Therefore we must have (see e.g. page 55 of [30])

$$
\widetilde{S}\left(M_{0}, V\right)=W^{-1}\left(\begin{array}{cc}
0 & -1 \\
1 & 0
\end{array}\right) W,
$$

\footnotetext{
${ }^{9}$ Via (4.2), these are related to fixed points $i$ and $e^{2 \pi i / 3}$ in the upper half plane under subgroups of $S L(2, \mathbb{Z})$.

${ }^{10}$ This follows from the fact that $-I_{2}$ in either $S L(2, \mathbb{Z})_{S}, S L(2, \mathbb{Z})_{T}$ or $S L(2, \mathbb{Z})_{U}$ changes the signs of all the charges and therefore changes the sign of the minor matrix. This is equivalent to multiplying the minor matrix by $-I_{4}$ from the right.
} 
for some $S L(2, \mathbb{Z})$ matrix $W$. In this case we can express (3.20) as

$$
W M_{0} V=\left(\begin{array}{cc}
0 & -1 \\
1 & 0
\end{array}\right) W M_{0} .
$$

If we now redefine $W M_{0}$ as the new $M_{0}$, then comparing (E.3) with (3.20) we get

$$
\widetilde{S}\left(M_{0}, V\right)=\left(\begin{array}{cc}
0 & -1 \\
1 & 0
\end{array}\right)
$$

Next we consider the case where one of the quadratic forms is proportional to $\left(x^{2}+x y+y^{2}\right)$. In this case, $W_{2}$ given in (D.4) (and its powers) is the symmetry of the quadratic form. As in the previous case, let $V^{\prime}$ be the $4 \times 4$ matrix that represents this symmetry by multiplying $M_{0}$ from the right. Since $W_{2}^{3}=-I_{2}$, we must have $V^{\prime 3}=-I_{4}$. It follows from repeated application of (3.20) that $\widetilde{S}\left(M_{0}, V^{\prime}\right)^{3}=-I_{2}$. Therefore either $\widetilde{S}\left(M_{0}, V^{\prime}\right)=-I_{2}$ or 30

$$
\widetilde{S}\left(M_{0}, V^{\prime}\right)=\widetilde{W}^{-1}\left(\begin{array}{cc}
1 & -1 \\
1 & 0
\end{array}\right) \widetilde{W}
$$

for some $S L(2, \mathbb{Z})$ matrix $\widetilde{W}$. We shall soon rule out the possibility that $\widetilde{S}\left(M_{0}, V^{\prime}\right)=-I_{2}$. Therefore (E.5) must hold. As before, we can redefine $\widetilde{W} M_{0}$ as our new $M_{0}$, and get,

$$
\widetilde{S}\left(M_{0}, V^{\prime}\right)=\left(\begin{array}{cc}
1 & -1 \\
1 & 0
\end{array}\right)
$$

It remains to show that we cannot have $S_{0}\left(M, V^{\prime}\right)=-I_{2}$. To prove this, assume the contrary, so that $(3.20)$ gives

$$
M_{0} V^{\prime}=-M_{0}
$$

Therefore the two rows of $M_{0}$ give two left eigenvectors of $V^{\prime}$ of eigenvalue -1 . However since $V^{\prime}$ represents the action of $W_{2}$ on the charge vectors, this will mean that the charge vectors have eigenvalue -1 under the action of $W_{2}$ - as an element of $S L(2, Z)_{S}$ if $q_{1} \propto\left(x^{2}+x y+y^{2}\right)$ and as an element of $S L(2, \mathbb{Z})_{U}$ for $q_{2} \propto\left(x^{2}+x y+y^{2}\right)$. However from the form of $W_{2}$ given in (D.4), one can easily see that it does not have an eigenvector with eigenvalue -1 . Therefore we cannot have $\widetilde{S}\left(M_{0}, V^{\prime}\right)=-I_{2}$.

Next consider the case where both $q_{1}$ and $q_{2}$ are proportional to $\left(x^{2}+y^{2}\right)$. Then they are invariant under $\left(W_{1}\right)_{S}$ and $\left(W_{1}\right)_{U}$ respectively. Let $V_{1}$ and $V_{2}$ be the $4 \times 4$ matrices, which, by multiplying $M_{0}$ from the right, generate the actions of $\left(W_{1}\right)_{S}$ and $\left(W_{1}\right)_{U}$ respectively. Since $\left(W_{1}\right)_{S}$ and $\left(W_{1}\right)_{U}$ commute, it follows that $V_{1}$ and $V_{2}$ commute. Now, following previous arguments, we can see that by appropriate choice of $M_{0}$, we can choose $\widetilde{S}\left(M_{0}, V_{1}\right)$ to be the 
matrix given in (E.4). Furthermore, since $V_{1}$ and $V_{2}$ commute, it follows by repeated application of (3.20) that $\widetilde{S}\left(M_{0}, V_{1}\right)$ and $\widetilde{S}\left(M_{0}, V_{2}\right)$ also commute. Also $\widetilde{S}\left(M_{0}, V_{2}\right)^{2}$ must be $-I_{2}$. It follows by explicit computation that $\widetilde{S}\left(M_{0}, V_{2}\right)$ is $\pm \widetilde{S}\left(M_{0}, V_{1}\right)$. Therefore $\widetilde{S}\left(M_{0}, V\right)$, with $V$ given by arbitrary products of powers of $V_{1}$ and $V_{2}$, will be given by the matrix given in (E.4) or its powers.

If both $q_{1}$ and $q_{2}$ are proportional to $\left(x^{2}+x y+y^{2}\right)$, then they are invariant under $\left(W_{2}\right)_{S}$ and $\left(W_{2}\right)_{U}$ respectively. Let $V_{3}$ and $V_{4}$ be the $4 \times 4$ matrices, which, by multiplying $M_{0}$ from the right, generate the actions of $\left(W_{2}\right)_{S}$ and $\left(W_{2}\right)_{U}$ respectively. As before, we can choose $M_{0}$ appropriately to make $\widetilde{S}\left(M_{0}, V_{3}\right)$ be given by the matrix appearing in (E.6). Since $\left(W_{2}\right)_{S}$ and $\left(W_{2}\right)_{U}$ commute, it follows that $V_{3}$ and $V_{4}$ commute and also $\widetilde{S}\left(M_{0}, V_{3}\right)$ and $\widetilde{S}\left(M_{0}, V_{4}\right)$ commute. A short computation, using the known form of $\widetilde{S}\left(M_{0}, V_{3}\right)$ given in (E.6), shows that $\widetilde{S}\left(M_{0}, V_{4}\right)$ must be given by some power of $\widetilde{S}\left(M_{0}, V_{3}\right)$. Therefore $\widetilde{S}\left(M_{0}, V\right)$, with $V$ given by arbitrary products of powers of $V_{3}$ and $V_{4}$, will also be given by powers of (E.6).

Since an overall minus sign in $V$ or $\widetilde{S}\left(M_{0}, V\right)$ can be absorbed into $S$ in (3.21), by examining the matrices given in (E.4), (E.6) and their powers, we see that we need to consider only three inequivalent choices of $\widetilde{S}\left(M_{0}, V\right)$ :

$$
\left(\begin{array}{cc}
0 & -1 \\
1 & 0
\end{array}\right), \quad\left(\begin{array}{cc}
1 & -1 \\
1 & 0
\end{array}\right), \quad\left(\begin{array}{cc}
0 & -1 \\
1 & -1
\end{array}\right) .
$$

The first one is relevant when either $q_{1}$ or $q_{2}$ is proportional to $\left(x^{2}+y^{2}\right)$, while the second and the third ones are relevant when either $q_{1}$ or $q_{2}$ is proportional to $\left(x^{2}+x y+y^{2}\right)$.

\section{F Counting duality orbits in different ways}

In our analysis in the text, we have treated the duality group $\Gamma_{T}$ differently from $\Gamma_{S}$ and $\Gamma_{U}$, in that we begin with a pair of quadratic forms representing equivalence classes of $\Gamma_{S}$ and $\Gamma_{U}$, and then find how many independent Bhargava cubes exist that reproduce these quadratic forms and yet cannot be related by $\Gamma_{S} \times \Gamma_{T} \times \Gamma_{U}$ transformations. However, we could exchange the roles of $\Gamma_{S}$ and $\Gamma_{T}$ or of $\Gamma_{U}$ and $\Gamma_{T}$ and carry out the counting. The total number of duality orbits counted in these different ways must be the same. In this section we shall verify this using several examples, We shall consider discriminants of the form $-4 k^{2}$ and $-3 k^{2}$ with $k \in \mathbb{Z}$, since for other discriminants there are no special quadratic forms that remain invariant under an element of $S L(2, \mathbb{Z})$, and in such cases the total number of duality orbits is given by 
the number of orbits of $S L(2, \mathbb{Z})^{3}$ times $n_{S} n_{T} n_{U}$ where $n_{S}, n_{T}$ and $n_{U}$ are the indices of $\Gamma_{S}$, $\Gamma_{T}$ and $\Gamma_{U}$ in $S L(2, \mathbb{Z})$. This is manifestly symmetric under permutations of $\Gamma_{S}, \Gamma_{T}$ and $\Gamma_{U}$. Also, to avoid repetition, we shall restrict the quadratic forms $q_{1}$ and $q_{2}$ to be positive definite. If we allow $q_{1}$ and $q_{2}$ to be of either sign, the total number of orbits will be given by four times what we shall count below, since the signs of $q_{1}$ and $q_{2}$ can be changed independently.

\section{F.1 $D=-16$ and duality group $S L(2, \mathbb{Z})_{S} \times \Gamma_{0}(2)_{T} \times S L(2, \mathbb{Z})_{U}$}

Counting 1 : We first carry out the analysis by letting $\Gamma_{0}(2)_{T}$ play the special role as in \$4. Therefore we begin by taking pairs of quadratic forms representing equivalence classes of $S L(2, \mathbb{Z})_{S}$ and $S L(2, \mathbb{Z})_{U}$. Possible inequivalent quadratic forms for $D=-16$ are

$$
f_{1}=x^{2}+4 y^{2} \quad \text { and } \quad f_{2}=2\left(x^{2}+y^{2}\right)
$$

Therefore, we have four possible pairs of $\left(q_{1}, q_{2}\right)$. We shall count the number of duality orbits for these four possible pairs. Note that among the two quadratic forms in ( $(\mathrm{F} .1), f_{2}$ is proportional to $f_{-4}$ and hence is invariant under $\operatorname{Aut}\left(f_{-4}\right)$ which is generated by $W_{1}$.

- Pair 1, $q_{1}=f_{1}, q_{2}=f_{1}$ : This pair is coprime, therefore the $U$ matrix introduced in (3.11) is the identity matrix. The pair also does not contain any special form. Therefore number of duality orbits is equal to number of representative elements of coset space $\Gamma_{0}(2)_{T} \backslash S L(2, \mathbb{Z})$ which is 3 . Hence, for pair 1 , we have 3 duality orbits.

- Pair 2, $q_{1}=f_{1}, q_{2}=f_{2}$ : This pair is also coprime, hence the $U$ matrix is again the identity matrix. However $q_{2}$ is a special form, and according to (3.22), we have $\widetilde{S}\left(M_{0}, V\right)=\left(\begin{array}{cc}0 & -1 \\ 1 & 0\end{array}\right)$. Therefore coset elements $\mathcal{G}$ and $\mathcal{G}^{\prime}$ of $\Gamma_{0}(2)_{T} \backslash S L(2, \mathbb{Z})$, related by $\mathcal{G}^{\prime}=\mathcal{G}\left(\begin{array}{cc}0 & -1 \\ 1 & 0\end{array}\right)$, describe the same duality orbit. It is now easy to see that this exchanges the first two coset representatives given in (4.14), leaving the third one unchanged. Hence we have 2 independent duality orbits for this pair.

- Pair 3, $q_{1}=f_{2}, q_{2}=f_{1}$ : The counting goes in the same way as for pair 2 and we get 2 independent duality orbits for this pair.

- Pair 4, $q_{1}=f_{2}, q_{2}=f_{2}$ : This pair is not coprime, since $m=\operatorname{gcd}\left(A_{1}, B_{1}, C_{1}, A_{2}, B_{2}, C_{2}\right)=$ 2. Hence the $U$ matrix defined in (3.11) can take three possible values:

$$
\left(\begin{array}{ll}
1 & 0 \\
0 & 2
\end{array}\right), \quad\left(\begin{array}{ll}
1 & 1 \\
0 & 2
\end{array}\right), \quad\left(\begin{array}{ll}
2 & 0 \\
0 & 1
\end{array}\right) .
$$


Each of these has three associated duality orbits from the three elements of the coset $\Gamma_{0}(2)_{T} \backslash S L(2, \mathbb{Z})$. However there will be further identification among these orbits since both the quadratic forms are special forms proportional to $f_{-4}$. According to (3.22), we have $\widetilde{S}\left(M_{0}, V\right)=\left(\begin{array}{cc}0 & -1 \\ 1 & 0\end{array}\right)$. It is easy to see that the first matrix in (F.2), multiplied by $\widetilde{S}\left(M_{0}, V\right)$ from the right, gives the last matrix in (F.2), multiplied by $-\widetilde{S}\left(M_{0}, V\right)$ from the left. The effect of the latter is to further exchange the coset elements of $\Gamma_{0}(2)_{T} \backslash S L(2, \mathbb{Z})$ as in the case of pair 3 . Therefore the three duality orbits associated with the first matrix in (F.2) are identified with the three duality orbits associated with the third matrix in $(\overline{F .2})$, and we get 3 independent orbits. On the other hand the second element of (F.2), multiplied by $\widetilde{S}\left(M_{0}, V\right)$ from the right, gives us back the second element multiplied by $\left(\begin{array}{ll}1 & -1 \\ 2 & -1\end{array}\right)$ from the left. Therefore we now need to check the effect of right multiplication of the coset elements (4.14) by this matrix. It is easy to check that this leaves invariant the first element of (4.14) and exchanges the second and the third elements. Therefore associated with the second element of (F.2) there are 2 duality orbits. This gives a total of 5 duality orbits for this pair.

Adding up all the numbers, we get a total of 12 duality orbits.

Counting 2: Now we let $S L(2, \mathbb{Z})_{U}$ play the special role that $\Gamma_{0}(2)_{T}$ played in the previous analysis. For this we begin with the pairs of inequivalent quadratic forms of $S L(2, \mathbb{Z})_{S}$ and $\Gamma_{0}(2)_{T}$. The inequivalent quadratic forms of $S L(2, \mathbb{Z})$ are already listed in (F.1). For $\Gamma_{0}(2)$ the inequivalent quadratic forms are:

$$
\begin{aligned}
& \tilde{f}_{1}=x^{2}+4 y^{2}, \quad \tilde{f}_{2}=2\left(x^{2}+y^{2}\right), \quad \tilde{f}_{3}=4 x^{2}+y^{2}, \\
& \tilde{f}_{4}=x^{2}+2 x y+5 y^{2}, \quad \tilde{f}_{5}=2\left(x^{2}+2 x y+2 y^{2}\right) .
\end{aligned}
$$

Of these $\tilde{f}_{5}$ is a special form that is invariant under the order 4 element $\left(\begin{array}{ll}1 & -1 \\ 2 & -1\end{array}\right)$ of $\Gamma_{0}(2)$. Therefore we have total 10 such pairs of $\left(q_{1}, q_{2}\right)$. We shall now count the number of duality orbits associated with each such pair.

1. The pairs $\left(f_{1}, \tilde{f}_{1}\right),\left(f_{1}, \tilde{f}_{2}\right),\left(f_{1}, \tilde{f}_{3}\right)$ and $\left(f_{1}, \tilde{f}_{4}\right)$ are coprime and do not contain any special form. Hence we have one duality orbit for each pair. This gives a total of 4 duality orbits from these 4 pairs.

2. $\left(f_{1}, \tilde{f}_{5}\right)$ pair is coprime and therefore $U$ is the identity matrix. Although it has one special 
form $\tilde{f}_{5}$, since the third duality group is $S L(2, \mathbb{Z})$, the special form plays no role and we have only 1 duality orbit for this pair.

3. The pairs $\left(f_{2}, \tilde{f}_{1}\right),\left(f_{2}, \tilde{f}_{3}\right)$ and $\left(f_{2}, \tilde{f}_{4}\right)$ are also coprime. They contain one special form $f_{2}$, but since the third duality group is $S L(2, \mathbb{Z})$, there is only one orbit for each pair, Therefore we get 3 duality orbits from these 3 pairs.

4. Next consider the pair $\left(f_{2}, \tilde{f}_{2}\right)$. This pair is not coprime and has $m=2$ as a common factor. Therefore $U$ has one of the three possible forms given in (F.2). Furthermore $f_{2}$ is special, being invariant under an order four duality transformation generated by $\left(\begin{array}{cc}0 & -1 \\ 1 & 0\end{array}\right)$. It then follows from (3.22) that by choosing $M_{0}$ appropriately, the corresponding $\widetilde{S}\left(M_{0}, V\right)$ can be taken to be $\left(\begin{array}{cc}0 & -1 \\ 1 & 0\end{array}\right)$. The analysis described below ( $\underline{F .2}$ ) shows that the orbits associated with the first and the third matrices in (F.2) must be identified. Therefore we have 2 duality orbits associated with this pair.

5. Finally consider the pair $\left(f_{2}, \tilde{f}_{5}\right)$. This pair is not coprime and has $m=2$ as a common factor. Furthermore both the forms are special forms, being invariant under order four elements of the duality groups. It follows from the arguments given below (4.13) that by suitably choosing $M_{0}$, we can pick $\widetilde{S}\left(M_{0}, V\right)$ to be the matrix $\left(\begin{array}{cc}0 & -1 \\ 1 & 0\end{array}\right)$. The analysis now reduces to the previous case, and gives a total of 2 duality orbits associated with this case.

Adding all the numbers, we get a total of 12 duality orbits. This agrees with the previous counting.

\section{F.2 $D=-16$ and duality group $\Gamma_{0}(2)_{S} \times S L(2, \mathbb{Z})_{T} \times \Gamma_{0}(2)_{U}$}

Counting 1 : Here we begin with a pair of quadratic forms $q_{1}$ and $q_{2}$ representing equivalence classes of $\Gamma_{0}(2)_{S}$ and $\Gamma_{0}(2)_{U}$, and classify the duality orbits for each such pair. There are 5 possible forms given in ( $(\mathrm{F.3})$, hence 25 possible pairs can be constructed.

1. Out of these 25 pairs 21 pairs are coprime. Since the third duality group is $S L(2, \mathbb{Z})$, these 21 coprime pairs give 21 orbits.

2. There is one pair $\left(\tilde{f}_{2}, \tilde{f}_{2}\right)$ which is non-coprime with gcd $=2$ and none of the forms are special. Therefore there are three $U$ matrices involved and hence this pair gives 3 orbits. 
3. There are two pairs $\left(\tilde{f}_{2}, \tilde{f}_{5}\right)$ and $\left(\tilde{f}_{5}, \tilde{f}_{2}\right)$ which are non-coprime with gcd $=2$ and both the pairs contain one special form $\tilde{f}_{5}$ that is invariant under an order four element of $\Gamma_{0}(2)$. Hence out of three $U$ matrices listed in $(\underline{\mathrm{F} .2})$, two will generate independent orbits. Therefore, each pair gives 2 orbits, and we get a total of 4 orbits.

4. Finally $\left(\tilde{f}_{5}, \tilde{f}_{5}\right)$ pair is non-coprime and both the forms are special, being invariant under an order four subgroup of $\Gamma_{0}(2)$. Hence out of three $U$ matrices two will be independent and hence this pair will give 2 orbits.

Thus this way of counting gives a total of 30 orbits.

Counting 2 : We now exchange the roles of $S L(2, \mathbb{Z})_{T}$ and $\Gamma_{0}(2)_{U}$. Therefore we begin with a pair of quadratic form $\left(q_{1}, q_{2}\right)$ representing equivalence classes of $S L(2, \mathbb{Z})_{T} \times \Gamma_{0}(2)_{S}$ and count the number of independent duality orbits for each pair. Using (F.1) and (F.3) we see that we have a total of 10 pairs of quadratic forms.

1. Out of these ten pairs, four are coprime and contain no special forms. Since the last duality group is $\Gamma_{0}(2)_{U}$ these four pairs generate $4 \times 3=12$ orbits.

2. $f_{1}, \tilde{f}_{5}$ are coprime but $\tilde{f}_{5}$ is a special form, invariant under an order four element of $\Gamma_{0}(2)$. The corresponding $\widetilde{S}$ matrices will relate two of the three $\mathcal{G}$ matrices listed in (4.14) to each other. Hence this pair will generate 2 orbits.

3. $f_{2}$ pairing with $\tilde{f}_{1}, \tilde{f}_{3}$ and $\tilde{f}_{4}$ gives coprime pairs but $f_{2}$ is a special form invariant under an order 4 element of $S L(2, \mathbb{Z})$. Therefore each of these three pairs will generate 2 orbits, giving a total of 6 orbits.

4. $\left(f_{2}, \tilde{f}_{2}\right)$ is non-coprime with gcd $=2$ and has a special form. This case is similar to that of Pair 4 in $\$$ F.1, and will generate 5 orbits.

5. $\left(f_{2}, \tilde{f}_{5}\right)$ is non-coprime with gcd $=2$ and both the forms are special forms. Again by arguments similar to that of Pair 4 in $\oint$ F.1, it will generate 5 orbits.

Adding up all the numbers, we get a total 30 orbits. This agrees with the previous counting. 


\section{F.3 $D=-36$ and duality group $S L(2, \mathbb{Z})_{S} \times S L(2, \mathbb{Z})_{T} \times \Gamma_{0}(2)_{U}$}

In this case the inequivalent quadratic forms of $S L(2, \mathbb{Z})$ are

$$
f_{1}=9 x^{2}+y^{2}, \quad f_{2}=2 x^{2}+2 x y+5 y^{2}, \quad f_{3}=3\left(x^{2}+y^{2}\right) .
$$

Of these $f_{3}$ is a special form, invariant under $W_{1}$. On the other hand the inequivalent quadratic forms of $\Gamma_{0}(2)$. are:

$\tilde{f}_{1}=x^{2}+9 y^{2}, \quad \tilde{f}_{2}=x^{2}+2 x y+10 y^{2}, \quad \tilde{f}_{3}=2 x^{2}+2 x y+5 y^{2}, \quad \tilde{f}_{4}=2 x^{2}-2 x y+5 y^{2}$,

$\tilde{f}_{5}=3\left(x^{2}+y^{2}\right), \quad \tilde{f}_{6}=3\left(x^{2}+2 x y+2 y^{2}\right), \quad \tilde{f}_{7}=5 x^{2}+2 x y+2 y^{2}, \quad \tilde{f}_{8}=9 x^{2}+y^{2}$.

In this list, $\tilde{f}_{6}$ is a special form, invariant under the order four element $\left(\begin{array}{ll}1 & -1 \\ 2 & -1\end{array}\right)$ of $\Gamma_{0}(2)$.

Counting 1 : Here we proceed as usual by taking a pair of quadratic forms $q_{1}$ and $q_{2}$, representing the equivalence classes of $S L(2, \mathbb{Z})_{S}$ and $\Gamma_{0}(2)_{U}$ respectively, and then find the number of orbits for each pair. There are altogether 24 such pairs.

1. There are 22 pairs of coprime forms. Each of these gives 1 orbit. Therefore we have 22 orbits from these 22 pairs.

2. The pair $\left(f_{3}, \tilde{f}_{5}\right)$ is non-coprime with $\mathrm{gcd}=3$ and contains one special form $f_{3}$. There are four $U$ matrices, given by:

$$
\left(\begin{array}{ll}
2 & 0 \\
0 & 1
\end{array}\right), \quad\left(\begin{array}{ll}
1 & 0 \\
0 & 2
\end{array}\right), \quad\left(\begin{array}{ll}
1 & 1 \\
0 & 2
\end{array}\right), \quad\left(\begin{array}{ll}
1 & 2 \\
0 & 2
\end{array}\right) .
$$

It is easy to see that right multiplication of these matrices by $\widetilde{S}=\left(\begin{array}{cc}0 & -1 \\ 1 & 0\end{array}\right)$ relates the first two of these matrices to each other and the last two of these matrices to each other, up to left multiplication by $S L(2, \mathbb{Z})$ matrices. Hence we have 2 independent $U$ matrices generating 2 orbits.

3. There is one non-coprime pair $\left(f_{3}, \tilde{f}_{6}\right)$ with gcd $=3$ and both the forms are special. The same argument as above tells us that we have 2 orbits for this pair.

This gives a total of 26 orbits. 
Counting 2 : We now take a pair of quadratic forms $\left(q_{1}, q_{2}\right)$ representing equivalence classes of $S L(2, \mathbb{Z})_{S}$ and $S L(2, \mathbb{Z})_{T}$, and count the duality orbits associated with each such pair. From (F.4) we see that we have 9 such pairs.

1. We have 4 coprime pairs with no special forms. Each of these gives 3 orbits associated with three elements of the coset $\Gamma_{0}(2)_{U} \backslash S L(2, Z)$ given in (4.14). This gives 12 orbits.

2. There are 4 coprime pairs with one special form. For each of these, the duality orbits generated by two of the three coset elements given in (4.14) get identified. Therefore we have $4 \times 2=8$ orbits.

3. We have one non-coprime pair $\left(f_{3}, f_{3}\right)$ with gcd $=3$ and both forms special. There are four $U$ matrices listed in ( $(\underline{\text { F.6 }})$, and we have already seen that right multiplication by $\widetilde{S}$ exchanges the first two elements of $(\underline{F .6})$ with each other and the last two elements of ( F.6 ) with each other. Therefore, irrespective of how this acts on the elements of the coset $\Gamma_{0}(2)_{U} \backslash S L(2, Z)$, the three orbits associated with the first element of (F.6) gets identified with the three orbits associated with the second element of (F.6), and the three orbits associated with the third element of (F.6) gets identified with the three orbits associated with the fourth element of (F.6). Therefore we have 6 orbits.

Adding the numbers we get 26 orbits, in agreement with the previous counting.

\section{F.4 $D=-12$ and duality group $S L(2, \mathbb{Z})_{S} \times \Gamma_{0}(2)_{T} \times S L(2, \mathbb{Z})_{U}$}

In this case the $S L(2, \mathbb{Z})$ inequivalent quadratic forms are

$$
f_{1}=x^{2}+3 y^{2}, \quad f_{2}=2\left(x^{2}+x y+y^{2}\right) .
$$

$f_{2}$ is a special form that is invariant under $W_{2}$. On the other hand, $\Gamma_{0}(2)$ inequivalent quadratic forms are:

$$
\tilde{f}_{1}=x^{2}+3 y^{2}, \quad \tilde{f}_{2}=x^{2}+2 x y+4 y^{2}, \quad \tilde{f}_{3}=2\left(x^{2}+x y+y^{2}\right), \quad \tilde{f}_{4}=3 x^{2}+y^{2} .
$$

As discussed in $\S 4$, there are no special forms among these.

Counting 1 : We take a pair of quadratic forms $\left(q_{1}, q_{2}\right)$ representing equivalence classes of $S L(2, \mathbb{Z})_{S}$ and $S L(2, \mathbb{Z})_{U}$ and count the number of duality orbits for each such pair. There are four such pairs. 
1. $\left(f_{1}, f_{1}\right)$ : These are coprime and none of the forms are special. Therefore the number of orbits is equal to number of elements of the coset $\Gamma_{0}(2)_{T} \backslash S L(2, \mathbb{Z})$, which is 3 .

2. $\left(f_{1}, f_{2}\right)$ and $\left(f_{2}, f_{1}\right)$ : These are coprime and one of the forms is special. Therefore we have two non-trivial $\widetilde{S}$ matrices given by the last two elements in (3.22). They cyclically permute the three $\mathcal{G}$ matrices listed in (4.14). Thus each pair gives one orbit and we have 2 orbits in total.

3. $\left(f_{2}, f_{2}\right)$ : These are non-coprime and have gcd $=2$. Furthermore both of them are special forms. Therefore we have three non-trivial $U$ matrices listed in ( $(\mathrm{F.2})$. It is straightforward to verify using (3.29) and (3.32) that the $\widetilde{S}$ matrices cyclically permute these three U matrices. Therefore, we have a total of 3 orbits coming from 3 independent $\mathcal{G}$ matrices listed in (4.14).

This gives a total of 8 orbits.

Counting 2: Here we pick $q_{1}, q_{2}$ to represent equivalence classes of $S L(2, \mathbb{Z})_{S}$ and $\Gamma_{0}(2)_{T}$ respectively and count the number of orbits associated with each pair. We have 8 such pairs.

1. There are four coprime pairs containing no special forms. These give 4 orbits.

2. $f_{2}$ paired with $\tilde{f}_{1}, \tilde{f}_{2}$ and $\tilde{f}_{4}$ give coprime pairs but each pair contains one special form $f_{2}$. These give 3 orbits.

3. $\left(f_{2}, \tilde{f}_{3}\right)$ is a non-coprime pair with gcd $=2$ and one special form $f_{2}$. The $\widetilde{S}$ matrices given by the last two elements in (3.22) will cyclically permute the three $U$-matrices in (F.2). Therefore we have 1 orbit.

Adding up the numbers we get 8 orbits, in agreement with the previous result.

\section{F.5 $D=-12$ and duality group $S L(2, \mathbb{Z})_{S} \times \Gamma_{0}(2)_{T} \times \Gamma_{0}(2)_{U}$}

All the relevant quadratic forms are given in eqs.(F.7), (F.8).

Counting 1 : First we construct the pairs taking a pair of quadratic forms representing equivalence classes $\Gamma_{0}(2)_{U}$ and $\Gamma_{0}(2)_{T}$. There are 16 such possible pairs with no special form. 
1. There are 15 coprime pairs and hence the $U$ matrix is identity. Since the third duality group is $S L(2, \mathbb{Z})_{S}$, these 15 coprime pairs give 15 orbits.

2. There is only one non-coprime pair with $\mathrm{gcd}=2$. There are three possible $U$ matrices (F.2) associated with this pair. Since the pair does not have any special form, the three $U$ matrices give independent orbits. Hence this pair generates 3 orbits.

Thus we get total 18 orbits.

Counting 2: We now take the first quadratic form of the pair from equivalence classes of $S L(2, \mathbb{Z})_{S}$ and the second one from equivalence classes of $\Gamma_{0}(2)_{U}$. Therefore there are 8 such pairs.

1. There are 4 coprime pairs with no special forms. Since the third duality group is $\Gamma_{0}(2)_{T}$, there are three representative $\mathcal{G}$ matrices for each pair. Hence we get total 12 orbits.

2. There are 3 coprime pairs with one special form. The special form generates two nontrivial $\widetilde{S}$ matrices, which cyclically permute the three representative $\mathcal{G}$ matrices. Hence, each pair will generate 1 orbit and we have total 3 orbits from these three pairs.

3. There is one non-coprime pair with gcd $=2$ and containing one special form. Therefore, for this pair we have three possible $U$ matrices and two non-trivial $\widetilde{S}$ matrices. These two $\widetilde{S}$ matrices will cyclically permute the three $U$ matrices and hence this pair will generate 3 orbits due to three different $\mathcal{G}$ matrices.

Therefore we have total 18 orbits, in agreement with the previous counting.

\section{References}

[1] G. W. Moore, "Attractors and arithmetic," arXiv:hep-th/9807056 [hep-th]].

[2] G. W. Moore, "Arithmetic and attractors," arXiv:hep-th/9807087 [hep-th]].

[3] G. W. Moore, "Strings and Arithmetic," doi:10.1007/978-3-540-30308-48 arXiv:hep-th/0401049 [hep-th]].

[4] N. Askitas, "Embeddings of 2-spheres in 4-manifolds," Manuscripta Math. 8935 (1996). 
[5] A. Dabholkar, D. Gaiotto and S. Nampuri, "Comments on the spectrum of CHL dyons," JHEP 01, 023 (2008) doi:10.1088/1126-6708/2008/01/023 |arXiv:hep-th/0702150 [hepth]].

[6] S. Banerjee and A. Sen, "S-duality Action on Discrete T-duality Invariants," JHEP 04, 012 (2008) doi:10.1088/1126-6708/2008/04/012 [arXiv:0801.0149 [hep-th]].

[7] S. Banerjee and A. Sen, "Duality orbits, dyon spectrum and gauge theory limit of heterotic string theory on $T^{6}$," JHEP 03, 022 (2008) doi:10.1088/1126-6708/2008/03/022 arXiv:0712.0043 [hep-th]].

[8] N. Benjamin, S. Kachru, K. Ono and L. Rolen, "Black holes and class groups," Research in the Mathematical Sciences. 2018;5 (4) :43 [arXiv:1807.00797 [math.NT]].

[9] M. Gunaydin, S. Kachru and A. Tripathy, "Black holes and Bhargava's invariant theory," arXiv:1903.02323 [hep-th]].

[10] F. Lemmermeyer, "Binary Quadratic Forms: An Elementary Approach to the Arithmetic of Elliptic and Hyperelliptic Curves," unpublished notes.

[11] M. Trifkovic, "Algebraic Theory of Quadratic Numbers," Springer (2013) doi:10.1007/9781-4614-7717-4.

[12] M. Bhargava, "Higher composition laws. I. A new view on Gauss composition, and quadratic generalizations," Ann. Math. 2 159(1), 217 ?250 (2004).

[13] A. Sen and C. Vafa, "Dual pairs of type II string compactification," Nucl. Phys. B 455, 165-187 (1995) doi:10.1016/0550-3213(95)00498-H [arXiv:hep-th/9508064 [hep-th]].

[14] K. Behrndt, R. Kallosh, J. Rahmfeld, M. Shmakova and W. K. Wong, "STU black holes and string triality," Phys. Rev. D 54, 6293-6301 (1996) doi:10.1103/PhysRevD.54.6293 arXiv:hep-th/9608059 [hep-th]].

[15] A. Gregori, C. Kounnas and P. Petropoulos, "Nonperturbative triality in heterotic and type II N=2 strings," Nucl. Phys. B 553, 108-132 (1999) doi:10.1016/S05503213(99)00281-3 [arXiv:hep-th/9901117 [hep-th]].

[16] A. Klemm and M. Marino, "Counting BPS states on the enriques Calabi-Yau," Commun. Math. Phys. 280, 27-76 (2008) doi:10.1007/s00220-007-0407-z arXiv:hep-th/0512227 [hep-th]]. 
[17] T. W. Grimm, A. Klemm, M. Marino and M. Weiss, "Direct Integration of the Topological String," JHEP 08, 058 (2007) doi:10.1088/1126-6708/2007/08/058 arXiv:hep-th/0702187 [hep-th]].

[18] G. Cardoso, B. de Wit and S. Mahapatra, "Exact results for an STU-model," JHEP 02, 127 (2020) doi:10.1007/JHEP02(2020)127 arXiv:1907.04077 [hep-th]].

[19] A. Sen, "A Twist in the Dyon Partition Function," JHEP 05, 028 (2010) doi:10.1007/JHEP05(2010)028 arXiv:0911.1563 [hep-th]].

[20] S. Ferrara, J. A. Harvey, A. Strominger and C. Vafa, "Second quantized mirror symmetry," Phys. Lett. B 361, 59-65 (1995) doi:10.1016/0370-2693(95)01074-Z arXiv:hep-th/9505162 [hep-th]].

[21] D. A. Buell, "Binary Quadratic Forms: Classical Theory and Modern Computations," Springer-Verlag (1989).

[22] J. Buchmann, U. Vollmer, "Binary quadratic forms. An algorithmic approach. Algorithms and Computation in Mathematics," 20. Springer, Berlin ( 2007).

[23] B. Cho, "On the Г-Eqivalence of Binary Quadratic Forms," arXiv:1711.00230[math-NT].

[24] L. Borsten, " $E_{7}(7)$ invariant measures of entanglement," Fortsch. Phys. 56 (2008) 842-848.

[25] L. Borsten, "Aspects of M-Theory and Quantum Information," PhD thesis, Imperial College, 2010.

[26] L. Borsten, M. J. Duff and A. Marrani, "Black Holes and Higher Composition Laws," arXiv:2006.03574 [hep-th]].

[27] H. Cohen, "A Course in Computational Algebraic Number Theory," Springer (2000).

[28] D. A. Cox, "Primes of the form $x^{2}+n y^{2}$," Wiley (2013).

[29] A. Sebbar, "Torsion-free Genus Zero Congruence Subgroups of $P S L(2, \mathbb{R})$, , Duke Mathematical Journal, 110, 377 (2001).

[30] F. Diamond and J, Shurman "A First Course in Modular Forms," Springer (2005). 